\title{
PDE-CONSTRAINED OPTIMIZATION SUBJECT TO POINTWISE CONSTRAINTS ON THE CONTROL, THE STATE, AND ITS DERIVATIVE*
}

\author{
MICHAEL HINTERMÜLLER ${ }^{\dagger}$ AND KARL KUNISCH ${ }^{\ddagger}$
}

\begin{abstract}
A general Moreau-Yosida-based framework for minimization problems subject to partial differential equations and pointwise constraints on the control, the state, and its derivative is considered. A range space constraint qualification is used to argue existence of Lagrange multipliers and to derive a KKT-type system for characterizing first-order optimality of the unregularized problem. The theoretical framework is then used to develop a semismooth Newton algorithm in function space and to prove its locally superlinear convergence when solving the regularized problems. Further, for maintaining the local superlinear convergence in function space it is demonstrated that in some cases it might be necessary to add a lifting step to the Newton framework in order to bridge an $L^{2}-L^{r}$-norm gap, with $r>2$. The paper ends by a report on numerical tests.
\end{abstract}

Key words. constraint qualification, gradient constraints, first-order optimality system, lifting, Moreau-Yosida regularization, PDE-constrained optimization, pointwise state constraints, semismooth Newton method

AMS subject classifications. 49M15, 49M37, 65K05, 90C33

DOI. $10.1137 / 080737265$

1. Introduction. In the late 1980s and early 1990s a number of research efforts focused on the existence of Lagrange multipliers for pointwise state constraints in optimal control of partial differential equations (PDEs); see, for instance, [4] in the case of zero-order state constraints, i.e., $\varphi \leq y \leq \psi$, and [5] for constraints on the gradient of $y$ such as $|\nabla y| \leq \psi$, as well as the references therein. Here, $y$ denotes the state of an underlying (system of) PDEs and $\varphi, \psi$ represent suitably chosen bounds. While $[4,5]$ focus on second-order linear elliptic differential equations and trackingtype objective functionals, subsequent work such as, e.g., [22, 23] considered parabolic PDEs and/or various types of nonlinearities. Moreover, investigations of second-order optimality conditions in the presence of pointwise state constraints can be found in [24] and the references therein. In many of these papers, for guaranteeing the existence of multipliers it is common to rely on the Slater constraint qualification, which requires that the feasible set contains an interior point.

Concerning the development of numerical solution algorithms for PDE-constrained optimization problems subject to pointwise state constraints, significant advances were obtained only in comparatively recent work. In [14, 9, 10], for instance, MoreauYosida-based inexact primal-dual path-following techniques are proposed and analyzed, and in $[17,20,27]$ Lavrentiev regularization is considered which replaces $y \leq \psi$ by the mixed constrained $\epsilon u+y \leq \psi$ with $u$ denoting the control variable and $\epsilon>0$ a

*Received by the editors October 5, 2008; accepted for publication (in revised form) April 18, 2009; published electronically August 19, 2009. Both authors acknowledge support by the Austrian Science Fundation FWF under SFB "Mathematical Optimization and Applications in Biomedical Sciences." M.H. further gratefully acknowledges support by the Austrian Ministry of Science and Education under START-program Y305 "Interfaces and Free Boundaries" administered by the FWF. http://www.siam.org/journals/siopt/20-3/73726.html

${ }^{\dagger}$ Department of Mathematics, Humboldt-University of Berlin, Unter den Linden 6, 10099 Berlin, Germany (hint@math.hu-berlin.de).

${ }^{\ddagger}$ Department of Mathematics and Scientific Computing, University of Graz, Heinrichstrasse 36, A-8010 Graz, Austria (karl.kunisch@uni-graz.at). 
small regularization parameter. In $[11,12]$ a technique based on shape sensitivity and level set methods is introduced. These works do not consider the case of combined control and state constraints and the case of pointwise constraints on the gradient of the state. Concerning the optimal control of ordinary differential equations with control as well as state constraints we mention $[3,16]$ and references given there. Control problems governed by PDEs with states and controls subject to pointwise constraints can be found, e.g., in $[1,6,15,19]$ and the references therein.

In the present paper we investigate the case where pointwise constraints on the control and the state variable appear simultaneously and independently, i.e., not linked as in the mixed case, which implies a certain extra regularity of the Lagrange multipliers. First- and second-order state constraints are admitted. To obtain efficient numerical methods, regularization of the state constraints is required. Here we investigate the Moreau-Yosida technique, which turns out to be very flexible with respect to various types of pointwise state constraints and can combined with pointwise constraints on the control variable, which need not be regularized. This flexibility makes it an ideal candidate for a unifying approach to a wide range of PDE-constrained minimization problems subject to pointwise constraints of controls and states with respect to both the proof of existence of Lagrange multipliers and the design of algorithms. Concerning the latter, we show in this paper that for the numerical solution of the associated subproblems semismooth Newton solvers are available which allow a function space analysis and converge locally at a $q$-superlinear rate. In addition, the path-following technique of [9] (see also [10]) provides an update tool for the regularization parameter, leading to efficient inexact path-following iterations. Further, for the proof of existence of multipliers, the Moreau-Yosida approach is based on a constraint qualification which is weaker than the usually invoked Slater condition. In [19] such a condition is used for pointwise zero-order state constraints.

The remainder of the paper is organized as follows. In section 2 we introduce the underlying rather general problem class, a constraint qualification of range-space-type and the Moreau-Yosida regularized problem. Moreover, the existence of multipliers for the unregularized problem is guaranteed and an associated first-order optimality characterization is derived. Section 3 is concerned with the semismooth Newton method for solving the regularized problems. It turns out that for a certain subclass of the underlying general problem a lifting step is necessary in order to bridge an $L^{2}$ - $L^{r}$-norm gap with $r>2$. The gap occurs due to the fact that the natural function space for the regularization is $L^{2}$ whereas the existence of multipliers requires $L^{r}$ regularity of the associated control variable. Here "lifting" refers to the fact that the standard semismooth Newton iteration has to be equipped with an additional step lifting the Newton updated from $L^{2}$ to $L^{r}$; see [28] for a related concept. Lifting, in the context of the present paper, is used for pointwise zero-order state constraints if the spatial dimension is larger than three, and for pointwise constraints on the gradient. Section 4 ends the paper by a report on numerical test. The appendix contains a chain rule result for the composition of two Newton differentiable functions, which is of interest in its own right.

2. Moreau-Yosida regularization and first-order optimality. In this section we derive, in a rather general setting and under a weak constraint qualification, first-order optimality conditions for the problem

$$
\left\{\begin{array}{l}
\operatorname{minimize} J(y, u)=J_{1}(y)+\frac{\alpha}{2}\left|u-u_{d}\right|_{L^{2}(\tilde{\Omega})}^{2} \\
\text { subject to } A y=E_{\tilde{\Omega}} u, \quad u \in C_{u}, \quad y \in C_{y},
\end{array}\right.
$$


where the control domain $\tilde{\Omega}$ is an open subset of $\Omega$, and the constraints on the control variable $u$ and the state variable $y$ are defined by

$$
C_{u}=\left\{u \in L^{2}(\tilde{\Omega}): \underline{\varphi} \leq u \leq \bar{\varphi} \text { a.e. in } \tilde{\Omega}\right\}, \quad C_{y}=\{y \in W:|G y| \leq \psi \text { in } \Omega\} .
$$

Here $A \in \mathcal{L}(W, L)$ with $W$ and $L$ reflexive Banach spaces of functions defined on the bounded domain $\Omega \subset \mathbb{R}^{d}$, satisfying $L^{r}(\Omega) \subset L$, with dense embedding, $2 \leq r<\infty$ and

$$
\left\langle v_{1}, v_{2}\right\rangle_{L^{*}, L}=\left(v_{1}, v_{2}\right)_{L^{r^{\prime}}(\Omega), L^{r}(\Omega)} \text { for all } v_{1} \in L^{*}, v_{2} \in L^{r}(\Omega),
$$

with $\frac{1}{r}+\frac{1}{r^{\prime}}=1$. Further $E_{\tilde{\Omega}}: L^{r}(\tilde{\Omega}) \rightarrow L^{r}(\Omega)$ is the extension-by-zero operator with adjoint $E_{\tilde{\Omega}}^{*}$ the restriction-to- $\tilde{\Omega}$ operator. The quantifiers characterizing the constraint sets $C_{u}$ and $C_{y}$ satisfy $G \in \mathcal{L}\left(W, \mathcal{C}(\bar{\Omega})^{l}\right)$ for some $1 \leq l \leq d$,

$$
\underline{\varphi}, \bar{\varphi} \in L^{2(r-1)}(\tilde{\Omega}), \text { and } \psi \in \mathcal{C}(\bar{\Omega}), 0<\underline{\psi} \leq \psi, \text { for some } \underline{\psi} \in \mathbb{R},
$$

$|\cdot|$ denotes the Euclidean-norm in $\mathbb{R}^{l}$ and the inequalities are interpreted in the pointwise almost everywhere (a.e.) sense. The minor extra regularity that is assumed by requiring that $\varphi, \bar{\varphi} \in L^{2(r-1)}(\tilde{\Omega})$ rather than $\varphi, \bar{\varphi} \in L^{2}(\tilde{\Omega})$ will be used in two ways: first the intermediate extra regularity $\underline{\varphi}, \bar{\varphi} \in L^{r}(\tilde{\Omega})$ is used for the sake of consistency with assumption (H4) below and, secondly, the $L^{2(r-1)}(\tilde{\Omega})$ bound on the admissible controls will be required for passing to the limit in a sequence of approximating problems to $(\mathrm{P})$ in Theorem 2.1 below.

The cost-functional is supposed to satisfy:

$$
\begin{aligned}
& J_{1} \in \mathcal{C}^{1,1}(W, \mathbb{R}) \text { is convex and } y_{n} \rightarrow y \text { in } W \text { implies that } \\
& J_{1}\left(y_{n}\right) \rightarrow J_{1}(y) \text { and } J_{1}^{\prime}\left(y_{n}\right) \rightarrow J_{1}^{\prime}(y) \text { in } W^{*} .
\end{aligned}
$$

Here and below " $\rightarrow$ " and " - " indicate strong and weak convergence, respectively. Moreover we fix

$$
\alpha>0 \text { and } u_{d} \in L^{2}(\tilde{\Omega}) .
$$

In addition to the above technical assumptions, we require the following hypotheses:

There exists a feasible point for the constraints in $(\mathrm{P})$.

$$
\begin{gathered}
A: W \rightarrow L \text { is a homeomorphism. } \\
G: W \rightarrow \mathcal{C}(\bar{\Omega})^{l} \text { is a compact linear operator. }
\end{gathered}
$$

$\left\{\begin{array}{l}\text { There exists a bounded set } M \subset C_{y} \times C_{u} \subset W \times L^{r}(\tilde{\Omega}) \text { such that } \\ 0 \in \operatorname{int}\left\{A y-E_{\tilde{\Omega}} u:(y, u) \in M\right\} \subset L^{r}(\Omega) \text {, where the interior is taken } \\ \text { with respect to } L^{r}(\Omega) .\end{array}\right.$

Conditions (H1) and (H2) are needed for existence of a solution to (P) and the hypotheses (H3)-(H4) are used to establish an optimality system. In particular, (H4) guarantees the existence of a Lagrange multiplier, or an adjoint state, associated with 
the equality constraint. Condition (H4) is weaker than Slater-type conditions. This can be argued as in [19, pp. 113-122]; see also [25]. In fact, let $(\bar{y}, \bar{u}) \in W \times L^{r}(\Omega)$ satisfy

$$
A \bar{y}=E_{\tilde{\Omega}} \bar{u}, \quad \underline{\varphi} \leq \bar{u} \leq \bar{\varphi}, \quad|G \bar{y}(\mathrm{x})|<\psi(\mathrm{x}) \text { for all } \mathrm{x} \in \bar{\Omega} .
$$

For $\rho>0$ and $|\eta|_{L^{r}(\Omega)} \leq \rho$ let $y_{\eta}$ denote the solution to

$$
A y_{\eta}=E_{\tilde{\Omega}} \bar{u}+\eta .
$$

Then

$$
\left|y_{\eta}-\bar{y}\right|_{W} \leq \rho\left\|A^{-1}\right\|_{\mathcal{L}\left(L^{r}(\Omega), W\right)} .
$$

Hence, if $\rho$ is sufficiently small, then $y_{\eta} \in C_{y}$ and the set

$$
M=\left\{\left(y_{\eta}, \bar{u}\right): \eta \in L^{r}(\Omega),|\eta|_{L^{r}(\Omega)} \leq \rho\right\}
$$

serves the purpose required by condition (H4). Differently from the Slater condition (2.5), condition (H4) operates in the range space of the operator $A$. Note also that when arguing that (2.5) implies (H4) the freedom to vary $u \in C_{u}$ was not used. For the analysis of the proposed algorithm it will be convenient to introduce the operator $B=G A^{-1} E_{\tilde{\Omega}}$. Conditions (H2) and (H3) imply that $B \in \mathcal{L}\left(L^{r}(\tilde{\Omega}), \mathcal{C}(\bar{\Omega})^{l}\right)$. The compactness assumption in (H3) is needed to pass to the limit in an appropriately defined approximation to problem $(\mathrm{P})$.

To argue existence for $(\mathrm{P})$, note that any minimizing sequence $\left\{\left(u_{n}, y\left(u_{n}\right)\right)\right\}$ is bounded in $L^{r}(\tilde{\Omega}) \times W$ by the properties of $C_{u}$ and (H2). The properties of $C_{u}$ as well as $C_{y}$, strict convexity of $J$ together with a subsequential limit argument guarantee the existence of a unique solution $\left(y^{*}, u^{*}\right)$ of $(\mathrm{P})$.

More general state constraints of the form

$$
C_{y}=\{\tilde{y} \in W:|(G \tilde{y})(\mathrm{x})-g(\mathrm{x})| \leq \psi \text { for all } \mathrm{x} \in \bar{\Omega}\}
$$

for some $g \in \mathcal{C}(\bar{\Omega})^{l}$ can be treated as well. In fact, if there exists $\tilde{y}_{g} \in W$ with $G y_{g}=g$ and $A y_{g} \in L^{r}(\Omega)$, then the shift $y:=\tilde{y}-y_{g}$ brings us back to the framework considered in (P) with a state equation of the form $A y=u-A y_{g}$, i.e., an affine term must be admitted and in (H4) the expression $A y-E_{\tilde{\Omega}} u$ must be replaced by $A y-E_{\tilde{\Omega}} u-A y_{g}$.

Before we establish first-order optimality, let us mention two problem classes that are covered by our definition of $C_{y}$ in $(\mathrm{P})$.

Example 1 (pointwise zero-order state constraints). Let $A$ denote the secondorder linear elliptic partial differential operator

$$
A y=-\sum_{i, j=1}^{d} \partial_{\mathrm{x}_{j}}\left(a_{i j} \partial_{\mathrm{x}_{i}} y\right)+a_{0} y
$$

with $C^{0, \delta}(\bar{\Omega})$-coefficients $a_{i j}, i, j=1, \ldots, d$, for some $\delta \in(0,1]$, which satisfy $\sum_{i, j=1}^{d} a_{i j}(\mathrm{x}) \xi_{i} \xi_{j} \geq \kappa|\xi|^{2}$ for almost all $\mathrm{x} \in \Omega$ and for all $\xi \in \mathbb{R}^{d}$, and $a_{0} \in L^{\infty}(\Omega)$ with $a_{0} \geq 0$ a.e. in $\Omega$. Here we have $\kappa>0$. The domain $\Omega$ is assumed to be either polyhedral and convex or to have a $\mathcal{C}^{1, \delta}$-boundary $\Gamma$ and to be locally on one side of 
$\Gamma$. We choose $W=W_{0}^{1, p}(\Omega), L=W^{-1, p}(\Omega), p>d$, and $G=$ id, which implies $l=1$. Then

$$
|G y| \leq \psi \text { in } \Omega \quad \Longleftrightarrow \quad-\psi \leq y \leq \psi \text { in } \Omega
$$

which is the case of zero-order pointwise state constraints. Since $p>d$, condition (H3) is satisfied. Moreover, $A: W \rightarrow W^{-1, p}(\Omega)$ is a homeomorphism [26, p. 179] so that, in particular, (H2) holds. Moreover there exists a constant $C$ such that

$$
|u|_{L} \leq C|u|_{L^{2}(\tilde{\Omega})}, \text { for all } u \in L^{2}(\tilde{\Omega})
$$

provided that $2 \leq \frac{d p}{d p-d-p}$. Consequently, we can take $r=2$. Here we use the fact that $W^{1, \frac{p}{p-1}}(\Omega)$ embeds continuously into $L^{2}(\Omega)$, provided that $2 \leq \frac{d p}{d p-d-p}$, and hence $L^{2}(\Omega) \subset W^{-1, p}(\Omega)$. Note that $2 \leq \frac{d p}{d p-d-p}$ combined with $d<p$ can only hold for $d \leq 3$.

In case $\Gamma$ is sufficiently regular so that $A$ is a homeomorphism from $H^{2}(\Omega) \cap$ $H_{0}^{1}(\Omega) \rightarrow L^{2}(\Omega)$, we can take $W=H^{2}(\Omega) \cap H_{0}^{1}(\Omega), L=L^{2}(\Omega)$, and $r=2$. In this case again (H2) and (H3) are satisfied if $d \leq 3$.

Example 2 (pointwise first-order state constraints). Let $A$ be as in (i) but with $C^{0,1}(\bar{\Omega})$-coefficients $a_{i j}$, and let $\Omega$ have a $\mathcal{C}^{1,1}$ boundary. Choose $W=W^{2, r}(\Omega) \cap$ $W_{0}^{1, r}(\Omega), L=L^{r}(\Omega), r>d$, and, for example, $G=\nabla$, which yields $l=d$. Then

$$
C_{y}=\{y \in W:|\nabla y(\mathrm{x})| \leq \psi(\mathrm{x}) \text { for all } \mathrm{x} \in \bar{\Omega}\}
$$

and (H2) and (H3) are satisfied due to the compact embedding of $W^{2, r}(\Omega)$ into $C^{1}(\bar{\Omega})$ if $r>d$.

An alternative treatment of pointwise first-order state constraints can be found in $[5]$.

If, on the other hand, $G=\mathrm{id}$, as in Example 1, then it suffices to choose $r \geq$ $\max \left(\frac{d}{2}, 2\right)$ for $(\mathrm{H} 2)$ and (H3) to hold.

We emphasize here that our notion of zero- and first-order state constraints does not correspond to the concept used in optimal control of ordinary differential equations. Rather, it refers to the order of the derivatives involved in the pointwise state constraints.

For deriving a first-order optimality system for $(\mathrm{P})$, we introduce the regularized problem

$\left(\mathrm{P}_{\gamma}\right) \quad\left\{\begin{array}{l}\operatorname{minimize} J_{1}(y)+\frac{\alpha}{2}\left|u-u_{d}\right|_{L^{2}(\tilde{\Omega})}^{2}+\frac{\gamma}{2}\left|(|G y|-\psi)^{+}\right|_{L^{2}(\Omega)}^{2} \\ \text { subject to } A y=E_{\tilde{\Omega}} u, u \in C_{u},\end{array}\right.$

where $\gamma>0$ and $(\cdot)^{+}=\max (0, \cdot)$ in the pointwise a.e. sense. In the following sections we shall see that $\left(\mathrm{P}_{\gamma}\right)$ is also useful for devising efficient numerical solution algorithms.

Let $\left(y_{\gamma}, u_{\gamma}\right) \in W \times L^{r}(\tilde{\Omega})$ denote the unique solution of $\left(\mathrm{P}_{\gamma}\right)$. Utilizing standard surjectivity techniques and (H2), we can argue the existence of Lagrange multipliers

$$
\left(p_{\gamma}, \bar{\mu}_{\gamma}, \underline{\mu}_{\gamma}\right) \in L^{*} \times L^{r^{\prime}}(\tilde{\Omega}) \times L^{r^{\prime}}(\tilde{\Omega}),
$$

Copyright $@$ by SIAM. Unauthorized reproduction of this article is prohibited. 
with $\frac{1}{r}+\frac{1}{r^{\prime}}=1$, such that

$\left(\mathrm{OS}_{\gamma}\right)$

$$
\left\{\begin{array}{l}
A y_{\gamma}=E_{\tilde{\Omega}} u_{\gamma}, \\
A^{*} p_{\gamma}+G^{*} \lambda_{\gamma}=-J_{1}^{\prime}\left(y_{\gamma}\right), \\
\alpha\left(u_{\gamma}-u_{d}\right)-E_{\tilde{\Omega}}^{*} p_{\gamma}+\bar{\mu}_{\gamma}-\underline{\mu}_{\gamma}=0, \\
\bar{\mu}_{\gamma} \geq 0, \quad u_{\gamma} \leq \bar{\varphi}, \quad \bar{\mu}_{\gamma}\left(u_{\gamma}-\bar{\varphi}\right)=0, \\
\underline{\mu}_{\gamma} \leq 0, \quad u_{\gamma} \geq \underline{\varphi}, \quad \underline{\mu}_{\gamma}\left(u_{\gamma}-\underline{\varphi}\right)=0, \\
\lambda_{\gamma}=\gamma\left(\left|G y_{\gamma}\right|-\psi\right)^{+} q_{\gamma}, \\
q_{\gamma}(\mathrm{x}) \in \begin{cases}\left\{\frac{G y_{\gamma}}{\left|G y_{\gamma}\right|}(\mathrm{x})\right\} & \text { if }\left|G y_{\gamma}(\mathrm{x})\right|>0, \\
\bar{B}(0,1)^{l} & \text { else },\end{cases}
\end{array}\right.
$$

where $\bar{B}(0,1)^{l}$ denotes the closed unit ball in $\mathbb{R}^{l}$. Above, $A^{*} \in \mathcal{L}\left(L^{*}, W^{*}\right)$ is the adjoint of $A$ and $G^{*}$ denotes the adjoint of $G$ as operator in $\mathcal{L}\left(W, L^{2}(\Omega)^{l}\right)$. Note that the expression for $\lambda_{\gamma}$ needs to be interpreted pointwise for every $\mathrm{x} \in \Omega$ and $\lambda_{\gamma}(\mathrm{x})=0$ if $\left|\left(G y_{\gamma}\right)(\mathrm{x})\right|-\psi(\mathrm{x}) \leq 0$. In particular this implies that $\lambda_{\gamma}$ is uniquely defined for every $\mathrm{x} \in \Omega$. Moreover, we have $\lambda_{\gamma} \in L^{2}(\Omega)^{l}$, in fact, $\lambda_{\gamma} \in \mathcal{C}(\Omega)^{l}$. The adjoint equation, which is the second equation in $\left(\mathrm{P}_{\gamma}\right)$, must be interpreted as

$$
\left\langle p_{\gamma}, A v\right\rangle_{L^{*}, L}+\left(\lambda_{\gamma}, G v\right)_{L^{2}(\Omega)}=-\left\langle J_{1}^{\prime}\left(y_{\gamma}\right), v\right\rangle_{W^{*}, W} \text { for any } v \in W,
$$

i.e., in the very weak sense. For later use we introduce the scalar factor of $\lambda_{\gamma}$ defined by

$$
\lambda_{\gamma}^{s}:=\frac{\gamma}{\left|G y_{\gamma}\right|}\left(\left|G y_{\gamma}\right|-\psi\right)^{+} \text {on }\left\{\left|G y_{\gamma}\right|>0\right\} \quad \text { and } \quad \lambda_{\gamma}^{s}:=0 \text { else. }
$$

This implies that

$$
\lambda_{\gamma}=\lambda_{\gamma}^{s} G y_{\gamma}
$$

The boundedness of the primal and dual variables is established next.

Lemma 2.1. Let (H1)-(H4) hold. Then the family

$$
\left\{\left(y_{\gamma}, u_{\gamma}, p_{\gamma}, \bar{\mu}_{\gamma}-\underline{\mu}_{\gamma}, \lambda_{\gamma}^{s}\right)\right\}_{\gamma \geq 1}
$$

is bounded in $W \times L^{r}(\tilde{\Omega}) \times L^{r^{\prime}}(\Omega) \times L^{r^{\prime}}(\tilde{\Omega}) \times L^{1}(\Omega)$.

Proof. Since $u_{\gamma} \in C_{u}$ for all $\gamma \geq 1$ we have by (H2) that

$$
\left\{\left(y_{\gamma}, u_{\gamma}\right)\right\}_{\gamma \geq 1} \text { is bounded in } W \times L^{r}(\tilde{\Omega}) .
$$

By (H3) the family $\left\{G y_{\gamma}\right\}_{\gamma \geq 1}$ is bounded in $\mathcal{C}(\bar{\Omega})^{l}$ as well. Henceforth let $C$ denote a generic constant independent of $\gamma \geq 1$. Let $(y, u) \in M$ be arbitrary. By $\left(\mathrm{OS}_{\gamma}\right)$

$$
\left\langle p_{\gamma}, A\left(y_{\gamma}-y\right)\right\rangle_{L^{*}, L}+\left(\lambda_{\gamma}, G\left(y_{\gamma}-y\right)\right)_{L^{2}(\Omega)}=-\left\langle J_{1}^{\prime}\left(y_{\gamma}\right), y_{\gamma}-y\right\rangle_{W^{*}, W}
$$


and

$$
\begin{aligned}
\left(\lambda_{\gamma}, G\left(y_{\gamma}-y\right)\right)_{L^{2}(\Omega)} & =\gamma\left(\left(\left|G y_{\gamma}\right|-\psi\right)^{+} q_{\gamma}, G y_{\gamma}-G y\right)_{L^{2}(\Omega)} \\
& =\gamma \int_{\Omega}\left(\left|G y_{\gamma}\right|-\psi\right)^{+}\left(\left|G y_{\gamma}\right|-\psi\right)+\gamma \int_{\Omega}\left(\left|G y_{\gamma}\right|-\psi\right)^{+}\left(\psi-q_{\gamma} \cdot G y\right) \\
& \geq \gamma \int_{\Omega}\left|\left(\left|G y_{\gamma}\right|-\psi\right)^{+}\right|^{2}+\gamma \int_{\Omega}\left(\left|G y_{\gamma}\right|-\psi\right)^{+}(\psi-|G y|) \\
& \geq \gamma\left|\left(\left|G y_{\gamma}\right|-\psi\right)^{+}\right|_{L^{2}(\Omega)}^{2} .
\end{aligned}
$$

Therefore

$$
\left\langle p_{\gamma}, A\left(y_{\gamma}-y\right)\right\rangle_{L^{*}, L}+\gamma\left|\left(\left|G y_{\gamma}\right|-\psi\right)^{+}\right|_{L^{2}(\Omega)}^{2} \leq-\left\langle J_{1}^{\prime}\left(y_{\gamma}\right), y_{\gamma}-y\right\rangle_{W^{*}, W}
$$

and

$$
\begin{aligned}
\left\langle p_{\gamma},-A y+E_{\tilde{\Omega}} u\right\rangle_{L^{*}, L}+\gamma\left|\left(\left|G y_{\gamma}\right|-\psi\right)^{+}\right|_{L^{2}(\Omega)}^{2} & \\
& \leq-\left\langle J_{1}^{\prime}\left(y_{\gamma}\right), y_{\gamma}-y\right\rangle_{W^{*}, W}+\left(p_{\gamma}, E_{\tilde{\Omega}}\left(u-u_{\gamma}\right)\right)_{L^{r^{\prime}}(\Omega), L^{r}(\Omega)} .
\end{aligned}
$$

The first term on the right-hand side is bounded since $\left\{y_{\gamma}\right\}_{\gamma \geq 1}$ is bounded in $W$ and $J_{1} \in \mathcal{C}^{1,1}(W, \mathbb{R})$, and the second term satisfies

$$
\begin{aligned}
\left(p_{\gamma}, E_{\tilde{\Omega}}\left(u-u_{\gamma}\right)\right)_{L^{r^{\prime}}(\Omega), L^{r}(\Omega)}= & \left(\alpha\left(u_{\gamma}-u_{d}\right)+\bar{\mu}_{\gamma}-\underline{\mu}_{\gamma}, u-u_{\gamma}\right)_{L^{r^{\prime}}(\tilde{\Omega}), L^{r}(\tilde{\Omega})} \\
\leq & C+\left(\bar{\mu}_{\gamma}, u-\bar{\varphi}+\bar{\varphi}-u_{\gamma}\right)_{L^{2}(\tilde{\Omega})} \\
& -\left(\underline{\mu}_{\gamma}, u-\underline{\varphi}+\underline{\varphi}-u_{\gamma}\right)_{L^{2}(\tilde{\Omega})} \leq C .
\end{aligned}
$$

Inserting these estimates into (2.9) and utilizing (H4) and (2.1) we have the existence of a constant $C$ independent of $\gamma$ such that

$$
\left\{\left|p_{\gamma}\right|_{L^{r^{\prime}}(\Omega)}\right\}_{\gamma \geq 1} \text { is bounded. }
$$

Integrating the third equation of $\left(\mathrm{OS}_{\gamma}\right)$ over $\left\{\mathrm{x}: \bar{\mu}_{\gamma}(\mathrm{x})>0\right\}$, we deduce that $\left\{\bar{\mu}_{\gamma}\right\}_{\gamma \geq 1}$ is bounded in $L^{r^{\prime}}(\tilde{\Omega})$. Similarly $\left\{\underline{\mu}_{\gamma}\right\}_{\gamma \geq 1}$ is bounded in $L^{r^{\prime}}(\tilde{\Omega})$. Finally we turn to estimate the scalar factor $\lambda_{\gamma}^{s}$. We have

$$
\begin{aligned}
\int_{\Omega} \lambda_{\gamma}^{s} & =\int_{\Omega} \frac{\gamma}{\left|G y_{\gamma}\right|}\left(\left|G y_{\gamma}\right|-\psi\right)^{+} \leq \int_{\Omega} \frac{\gamma}{\frac{\psi^{2}}{1}}\left(\left|G y_{\gamma}\right|-\psi\right)^{+}\left|G y_{\gamma}\right| \\
& =-\frac{1}{\underline{\psi}^{2}}\left(p_{\gamma}, A y_{\gamma}\right)_{L^{r^{\prime}}(\Omega), L^{r}(\Omega)}-\frac{1}{\underline{\psi}^{2}}\left\langle J^{\prime}\left(y_{\gamma}\right), y_{\gamma}\right\rangle_{W^{*}, W} \leq C,
\end{aligned}
$$

where we used that $\lambda_{\gamma}^{s}=0$ on $\left\{\left|G y_{\gamma}\right| \leq \underline{\psi}\right\}$ and $\left|A y_{\gamma}\right|_{L^{r}(\Omega)}=\left|E_{\tilde{\Omega}} u_{\gamma}\right|_{L^{r}(\Omega)} \leq C$. Hence, $\left\{\lambda_{\gamma}^{s}\right\}_{\gamma \geq 1}$ is bounded in $L^{1}(\Omega)$.

The preceding lemma implies that there exists

$$
\left(y_{*}, u_{*}, p_{*}, \bar{\mu}_{*}, \underline{\mu}_{*}, \lambda_{*}^{s}\right) \in W \times L^{r}(\tilde{\Omega}) \times L^{r^{\prime}}(\tilde{\Omega}) \times L^{r^{\prime}}(\tilde{\Omega}) \times L^{r^{\prime}}(\tilde{\Omega}) \times \mathcal{M}(\bar{\Omega})=: X,
$$

where $\mathcal{M}(\bar{\Omega})$ are the regular Borel measures on $\bar{\Omega}$, such that on subsequence

$$
\left(y_{\gamma}, u_{\gamma}, p_{\gamma}, \bar{\mu}_{\gamma}, \underline{\mu}_{\gamma}, \lambda_{\gamma}^{s}\right) \rightarrow\left(y_{*}, u_{*}, p_{*}, \bar{\mu}_{*}, \underline{\mu}_{*}, \lambda_{*}^{s}\right) \text { in } X,
$$

Copyright (c) by SIAM. Unauthorized reproduction of this article is prohibited. 
which, for the $\lambda^{s}$ component, means that

$$
\int_{\Omega} \lambda_{\gamma}^{s} v \rightarrow \int_{\Omega} \lambda_{*}^{s} v \text { for all } v \in \mathcal{C}(\bar{\Omega}) .
$$

By (H3) this implies that

$$
G y_{\gamma} \rightarrow G y_{*} \text { in } \mathcal{C}(\bar{\Omega})^{l}
$$

and hence

$$
\int_{\Omega} \lambda_{\gamma} v \rightarrow\left\langle\lambda_{*}^{s}\left(G y_{*}\right), v\right\rangle_{\mathcal{M}, \mathcal{C}(\bar{\Omega})^{l}} \text { for all } v \in \mathcal{C}^{l}(\bar{\Omega})^{l} .
$$

Passing to the limit in the second equation of $\left(\mathrm{OS}_{\gamma}\right)$ we find

$$
\left(p_{*}, A v\right)_{L^{r^{\prime}}(\Omega), L^{r}(\Omega)}=-\left\langle\lambda_{*}^{s}\left(G y_{*}\right), G v\right\rangle_{\mathcal{M}^{l}(\bar{\Omega}), \mathcal{C}^{l}(\bar{\Omega})}-\left\langle J_{1}^{\prime}\left(y_{*}\right), v\right\rangle_{W^{*}, W}
$$

for all $v \in D_{A}$, where $D_{A}=\left\{v \in W: A v \in L^{r}(\Omega)\right\}$ and $\langle\cdot, \cdot\rangle_{\mathcal{M}^{l}(\bar{\Omega}), \mathcal{C}^{l}(\bar{\Omega})}$ denotes the duality pairing between $\mathcal{C}(\bar{\Omega})^{l}$ and $\mathcal{M}(\bar{\Omega})^{l}$, the space of regular vector-valued Borel measures on $\bar{\Omega}$. Since $D_{A}$ is dense in $W$ and since the right-hand side uniquely defines a continuous linear functional on $W$, a density argument implies that $p_{*}$ can be uniquely extended to an element in $L^{*}$, and the left-hand side can be replaced by $\left\langle p_{*}, A v\right\rangle_{L^{*}, L}$, for all $v \in W$.

We can now pass to the limit in the first three equations of $\left(\mathrm{OS}_{\gamma}\right)$ to obtain

$$
\begin{aligned}
& A y_{*}=E_{\tilde{\Omega}} u_{*} \quad \text { in } L^{r^{\prime}}(\Omega), \\
& \left\langle p_{*}, A v\right\rangle_{L^{*}, L}+\left\langle\lambda_{*}^{s}\left(G y_{*}\right), G v\right\rangle_{\mathcal{M}^{l}(\bar{\Omega}), \mathcal{C}^{l}(\bar{\Omega})}=-\left\langle J_{1}^{\prime}\left(y_{*}\right), v\right\rangle_{W^{*}, W} \text { for all } v \in W, \\
& \alpha\left(u_{*}-u_{d}\right)-E_{\tilde{\Omega}}^{*} p_{*}+\left(\bar{\mu}_{*}-\underline{\mu}_{*}\right)=0 \quad \text { in } L^{r^{\prime}}(\Omega) .
\end{aligned}
$$

Standard arguments yield

$$
\underline{\mu}_{*} \geq 0, \bar{\mu}_{*} \geq 0, \underline{\varphi} \leq u_{*} \leq \bar{\varphi} \quad \text { a.e. in } \tilde{\Omega} .
$$

Note that

$$
J_{1}\left(y_{\gamma}\right)+\frac{\alpha}{2}\left|u_{\gamma}-u_{d}\right|_{L^{2}(\tilde{\Omega})}^{2}+\frac{\gamma}{2}\left|\left(\left|G y_{\gamma}\right|-\psi\right)^{+}\right|_{L^{2}(\Omega)}^{2} \leq J_{1}\left(y^{*}\right)+\frac{\alpha}{2}\left|u^{*}-u_{d}\right|_{L^{2}(\tilde{\Omega})}^{2} .
$$

This implies that $\left|G y_{*}(\mathrm{x})\right| \leq \psi(\mathrm{x})$ for all $\mathrm{x} \in \Omega$, and hence $\left(y_{*}, u_{*}\right)$ is feasible. Moreover, by $(2.3)$

$$
\begin{aligned}
J_{1}\left(y_{*}\right)+\frac{\alpha}{2}\left|u_{*}-u_{d}\right|_{L^{2}(\tilde{\Omega})}^{2} & \leq \lim _{\gamma \rightarrow \infty} J_{1}\left(y_{\gamma}\right)+\lim \sup _{\gamma \rightarrow \infty} \frac{\alpha}{2}\left|u_{\gamma}-u_{d}\right|_{L^{2}(\tilde{\Omega})}^{2} \\
& \leq J_{1}\left(y^{*}\right)+\frac{\alpha}{2}\left|u^{*}-u_{d}\right|_{L^{2}(\tilde{\Omega})}^{2} .
\end{aligned}
$$

Since $\left(y_{*}, u_{*}\right)$ is feasible and the solution of $(\mathrm{P})$ is unique, it follows that $\left(y_{*}, u_{*}\right)=$ $\left(y^{*}, u^{*}\right)$. Moreover, from (2.15) and weak lower semicontinuity of norms we have that $\lim _{\gamma \rightarrow \infty} u_{\gamma}=u^{*}$ in $L^{2}(\tilde{\Omega})$. From $u_{\gamma} \in C_{u}$ for all $\gamma$, and $u^{*} \in C_{u}$, with $C_{u} \subset$ $L^{2(r-1)}(\tilde{\Omega})$ by $(2.2)$, together with Hölder's inequality we obtain

$$
\left|u_{\gamma}-u^{*}\right|_{L^{r}(\tilde{\Omega})} \leq\left|u_{\gamma}-u^{*}\right|_{L^{2}(\tilde{\Omega})}^{1 / r}\left|u_{\gamma}-u^{*}\right|_{L^{2(r-1)}(\tilde{\Omega})}^{(r-1) / r} \leq C\left|u_{\gamma}-u^{*}\right|_{L^{2}(\tilde{\Omega})}^{1 / r} \stackrel{\gamma \rightarrow \infty}{\longrightarrow} 0
$$

with some positive constant $C$. This yields the strong convergence of $u_{\gamma}$ in $L^{r}(\tilde{\Omega})$.

Copyright (c) by SIAM. Unauthorized reproduction of this article is prohibited. 
Complementary slackness of $u_{*}, \bar{\mu}_{*}$ and $\underline{\mu}_{*}$, i.e.,

$$
\bar{\mu}_{*}\left(u_{*}-\bar{\varphi}\right)=0, \quad \underline{\mu}_{*}\left(u_{*}-\underline{\varphi}\right)=0 \quad \text { a.e. in } \tilde{\Omega}
$$

now follows from the fourth and fifth equations of $\left(\mathrm{OS}_{\gamma}\right)$, respectively, the weak convergence of $\left(\bar{\mu}_{\gamma}, \underline{\mu}_{\gamma}\right)$ to $\left(\bar{\mu}_{*}, \underline{\mu}_{*}\right)$ in $L^{r^{\prime}}(\tilde{\Omega})^{2}$, and the strong convergence of $u_{\gamma}$ to $u_{*}$ in $L^{r}(\tilde{\Omega})$.

Let $y \in C_{y}$. Then $\int_{\Omega} \lambda_{\gamma}^{s}(|G y|-\psi) \leq 0$ and hence

$$
\int_{\Omega} \lambda_{*}^{s}(|G(y)|-\psi) \leq 0 .
$$

Moreover, $\int_{\Omega} \lambda_{*}^{s} \varphi \geq 0$ for all $\varphi \in \mathcal{C}(\bar{\Omega})$ with $\varphi \geq 0$.

For every accumulation point $\lambda_{*}^{s}$, the corresponding adjoint variable $p_{*}$ and Lagrange multipliers $\bar{\mu}_{*}, \bar{\mu}_{*}$ are unique. In fact, since $y_{*}=y^{*}$ is unique, the difference $\delta p$ of two accumulation points of $p_{\gamma}$ satisfies

$$
(\delta p, A v)=0 \quad \text { for all } v \in W,
$$

and since $A$ is a homeomorphism we have that $\delta p=0$.

From (2.12) we deduce that

$$
\bar{\mu}_{*}=\left(E_{\tilde{\Omega}}^{*} p_{*}-\alpha\left(u^{*}-u_{d}\right)\right)^{+}, \underline{\mu}_{*}=\left(E_{\tilde{\Omega}}^{*} p_{*}-\alpha\left(u^{*}-u_{d}\right)\right)^{-},
$$

where $(\cdot)^{-}=\min (0, \cdot)$ in the pointwise a.e. sense.

We summarize our above findings in the following theorem which provides necessary and sufficient first-order optimality conditions for $(\mathrm{P})$.

TheOREm 2.1. Let (2.1)-(2.4) and (H1)-(H4) hold. Then there exists

$$
\left(p_{*}, \bar{\mu}_{*}, \underline{\mu}_{*}, \lambda_{*}^{s}\right) \in L^{*} \times L^{r^{\prime}}(\tilde{\Omega}) \times L^{r^{\prime}}(\tilde{\Omega}) \times \mathcal{M}(\bar{\Omega})
$$

such that

$$
\begin{aligned}
A y^{*} & =E_{\tilde{\Omega}} u^{*} & & \text { in } L^{r}(\Omega), \\
A^{*} p_{*}+G^{*}\left(\lambda_{*}^{s} G y^{*}\right) & =-J_{1}^{\prime}\left(y^{*}\right) & & \text { in } W^{*}, \\
\alpha\left(u^{*}-u_{d}\right)-E_{\tilde{\Omega}}^{*} p_{*}+\left(\bar{\mu}_{*}-\underline{\mu}_{*}\right) & =0 & & \text { in } L^{r^{\prime}}(\tilde{\Omega}), \\
\bar{\mu}_{*} \geq 0, u^{*} \leq \bar{\varphi}, \bar{\mu}_{*}\left(u^{*}-\bar{\varphi}\right) & =0 & & \text { a.e. in } \tilde{\Omega}, \\
\underline{\mu}_{*} \geq 0, u^{*} \geq \underline{\varphi}, \underline{\mu}_{*}\left(u^{*}-\underline{\varphi}\right) & =0 & & \text { a.e. in } \tilde{\Omega},
\end{aligned}
$$

and $\int_{\Omega} \lambda_{*}^{s} \varphi \geq 0$ for all $\varphi \in \mathcal{C}(\bar{\Omega})$ with $\varphi \geq 0$. Further $\left(p_{\gamma}, \bar{\mu}_{\gamma}, \underline{\mu}_{\gamma}\right)$ converges weakly in $L^{r^{\prime}}(\Omega) \times L^{r^{\prime}}(\tilde{\Omega}) \times L^{r^{\prime}}(\tilde{\Omega})$ (along a subsequence) to $\left(p_{*}, \bar{\mu}_{*}, \underline{\mu}_{*}\right),\left\langle\lambda_{\gamma}^{s}, v\right\rangle \rightarrow\left\langle\lambda_{*}^{s}, v\right\rangle$ (along a subsequence) for all $v \in \mathcal{C}(\bar{\Omega})$, and $\left(y_{\gamma}, u_{\gamma}\right) \rightarrow\left(y^{*}, u^{*}\right)$ strongly in $W \times L^{r}(\tilde{\Omega})$ as $\gamma \rightarrow \infty$.

We briefly revisit the examples 1 and 2 in order to discuss the structure of the respective adjoint equation.

Example 1 (revisited). For the case of pointwise zero-order state constraints with $W=W_{0}^{1, p}(\Omega)$ the adjoint equation in variational form is given by

$$
\left\langle p_{*}, A v\right\rangle_{W_{0}^{1, p^{\prime}}(\Omega), W^{-1, p}(\Omega)}+\left\langle\lambda_{*}^{s} y^{*}, v\right\rangle_{\mathcal{M}(\bar{\Omega}), \mathcal{C}(\bar{\Omega})}=-\left\langle J_{1}^{\prime}\left(u^{*}\right), v\right\rangle_{W^{*}, W}
$$

for all $v \in W$.

Copyright $@$ by SIAM. Unauthorized reproduction of this article is prohibited. 
Example 2 (revisited). Returning to pointwise gradient constraints expressed by $|\nabla y(\mathrm{x})| \leq \psi(\mathrm{x})$ the adjoint equation can be expressed as

$$
\left\langle p_{*}, A v\right\rangle_{L^{r^{\prime}}(\Omega), L^{r}(\Omega)}+\left\langle\lambda_{*}^{s} \nabla y^{*}, \nabla v\right\rangle_{\mathcal{M}(\bar{\Omega})^{l}, \mathcal{C}(\bar{\Omega})^{l}}=-\left\langle J_{1}^{\prime}\left(u^{*}\right), v\right\rangle_{W^{*}, W}
$$

for all $v \in W=W^{2, r}(\Omega) \cap W_{0}^{1, r}(\Omega)$.

Remark 2.1. Condition (H4) is quite general and also allows the case $\psi=0$ on parts of $\Omega$. Here we only briefly consider a special case of such a situation. Let $\tilde{\Omega}=\Omega, r=2, L=L^{2}(\Omega), W=H^{2}(\Omega) \cap H_{0}^{1}(\Omega)$, and $G=I$; i.e. we consider zero-order state constraints without constraints on the controls, in dimensions $d \leq 3$. We assume that $A: W \rightarrow L^{2}(\Omega)$ is a homeomorphism and that (2.2) is replaced by

$$
0 \leq \psi, \psi \in \mathcal{C}(\bar{\Omega})
$$

In this case (H1), (H2), and (H4) are trivially satisfied and $C_{y}=\{y \in W:|y| \leq$ $\psi$ in $\Omega$ \}. The optimality system is given by

$\left(\mathrm{OS}_{\gamma}^{\prime}\right)$

$$
\left\{\begin{array}{l}
A y_{\gamma}=u_{\gamma}, \\
A^{*} p_{\gamma}+\lambda_{\gamma}=-J_{1}^{\prime}\left(y_{\gamma}\right), \\
\alpha\left(u_{\gamma}-u_{d}\right)-p_{\gamma}=0, \\
\lambda_{\gamma}=\gamma\left(\left|y_{\gamma}\right|-\psi\right)^{+} q_{\gamma}, \\
q_{\gamma}(\mathrm{x}) \in \begin{cases}\left\{\frac{y_{\gamma}}{y_{\gamma} \mid}(\mathrm{x})\right\} & \text { if }\left|y_{\gamma}(\mathrm{x})\right|>0, \\
\bar{B}(0,1) & \text { else },\end{cases}
\end{array}\right.
$$

with $\left(y_{\gamma}, u_{\gamma}, p_{\gamma}, \lambda_{\gamma}\right) \in W \times L^{2}(\Omega) \times L^{2}(\Omega) \times L^{2}(\Omega)$. As in Lemma 2.1 we argue, using (H4), that $\left\{\left(y_{\gamma}, u_{\gamma}, p_{\gamma}\right)\right\}_{\gamma \geq 1}$ is bounded in $W \times L^{2}(\Omega) \times W^{*}$. Since we do not assume that $\underline{\psi}>0$, we argue differently than before to obtain a bound on $\left\{\lambda_{\gamma}\right\}_{\gamma \geq 1}$. In fact, the second equation in $\left(\operatorname{OS}_{\gamma}^{\prime}\right)$ implies that $\left\{\lambda_{\gamma}\right\}_{\gamma \geq 1}$ is bounded in $W^{*}$. Hence there exists $\left(y^{*}, u^{*}, p_{*}, \lambda_{*}\right) \in W \times L^{2}(\Omega) \times L^{2}(\Omega) \times W^{*}$, such that $\left(y_{\gamma}, u_{\gamma}\right) \rightarrow\left(y^{*}, u^{*}\right)$ in $W \times L^{2}(\Omega)$ and $\left(p_{\gamma}, \lambda_{\gamma}\right) \rightarrow\left(p_{*}, \lambda_{*}\right)$ weakly in $L^{2}(\Omega) \times W^{*}$, as $\gamma \rightarrow \infty$. Differently from the case with state and control constraints, we have convergence of the whole sequence, rather than subsequential convergence of $\left(p_{\gamma}, \lambda_{\gamma}\right)$ in this case. In fact, the third equation in $\left(\operatorname{OS}_{\gamma}^{\prime}\right)$ implies the convergence of $p_{\gamma}$, and the second equation the convergence of $\lambda_{\gamma}$. Passing to the limit as $\gamma \rightarrow \infty$ we obtain from $\left(\operatorname{OS}_{\gamma}^{\prime}\right)$

$$
\left\{\begin{aligned}
A y^{*} & =u^{*}, \\
A^{*} p_{*}+\lambda_{*} & =-J_{1}^{\prime}\left(y^{*}\right), \\
\alpha\left(u^{*}-u_{d}\right)-p_{*} & =0,
\end{aligned}\right.
$$

and $\lambda_{*}$ has the additional properties as the limit of elements $\lambda_{\gamma}$. For example, if $\psi \geq \underline{\psi}>0$ on a subset $\hat{\Omega} \subset \Omega$ and $y^{*}$ is inactive on $\hat{\Omega}$, then $\lambda_{*}=0$ as functional on continuous functions with compact support in $\hat{\Omega}$.

3. Semismooth Newton method for $\left(\mathbf{P}_{\gamma}\right)$. As mentioned earlier, $\left(\mathrm{P}_{\gamma}\right)$ is appealing as it can be solved with superlinearly convergent numerical methods. Combined with a suitable update strategy for $\gamma$, an overall solution algorithm for $(\mathrm{P})$ is obtained. Here we analyze in detail the superlinear solution process of $\left(\mathrm{P}_{\gamma}\right)$, for a fixed value $\gamma$. The constants in this section therefore depend on $\gamma$. For the path-following strategy with respect to $\gamma$ one may proceed as in $[9,10]$.

Copyright $@$ by SIAM. Unauthorized reproduction of this article is prohibited. 
3.1. Newton differentiability/semismoothness. In [8], see also [7], for a mapping $F: \mathcal{X} \rightarrow \mathcal{Y}$, with $\mathcal{X}$ and $\mathcal{Y}$ Banach spaces, a generalized derivative is introduced in such a way that q-superlinear convergence of the Newton algorithm can be guaranteed without requiring that $F$ is Frechet differentiable. In fact, $F$ is called Newton (or slant) differentiable in an open set $U \subset \mathcal{X}$ if there exists a family of generalized derivatives $G_{F}(x) \in \mathcal{L}(\mathcal{X}, \mathcal{Y}), x \in U$, such that

$$
\lim _{|h|_{\mathcal{X} \rightarrow 0}}|h|_{\mathcal{X}}^{-1}\left|F(x+h)-F(x)-G_{F}(x+h) h\right|_{\mathcal{Y}}=0 \quad \text { for every } x \in U .
$$

Note that $F$ need not be Frechet-differentiable in order to have the property (3.1). In general, there exists a set of Newton derivatives at $x$ which becomes a singleton whenever $F$ is Frechet-differentiable at $x$. We also point out that (3.1) resembles the concept of semismoothness of a mapping, which was introduced in [18] for scalarvalued functionals on $\mathbb{R}^{n}$ and extended to the vector-valued case in [21]. The concept of semismoothness in finite dimensions, however, is linked to Rademacher's theorem, which states that locally Lipschitz continuous functions are almost everywhere differentiable. This concept is not available in infinite dimensions. But property (3.1) quantifies one of the essential ingredients for the Newton method to be locally superlinearly convergent. Consequently it is becoming customary now to refer to the Newton method, in infinite dimensions, as a semismooth Newton method, if (3.1) holds. As usual the Newton method for finding $x^{*} \in \mathcal{X}$ such that $F\left(x^{*}\right)=0$ consists in the iteration:

Algorithm 1 (semismooth Newton method).

(i) Choose $x^{0} \in \mathcal{X}$.

(ii) Unless some stopping rule is satisfied, perform the update step

$$
x^{k+1}=x^{k}-G_{F}\left(x^{k}\right)^{-1} F\left(x^{k}\right) \quad \text { for } k=0,1 \ldots
$$

This iteration is locally q-superlinearly convergent to $x^{*}$ within a neighborhood $U\left(x^{*}\right)$, if $x_{0} \in U\left(x^{*}\right)$, and (3.1) as well as

$$
\left\|G_{F}(x)^{-1}\right\|_{\mathcal{L}(\mathcal{Y}, \mathcal{X})} \leq C, \text { for a constant } C \text { independently of } x \in U\left(x^{*}\right),
$$

hold $[7,8]$.

The remainder of this subsection is devoted to the analysis of the semismoothness property (3.1) of the mapping $F_{\gamma}$, which defines the Newton iteration associated with $\left(\mathrm{OS}_{\gamma}\right)$. This is done for $\mathcal{X}=\mathcal{Y}=L^{r}(\tilde{\Omega})$ where the choice of $r$ is dictated by the need that $G y(u) \in \mathcal{C}(\bar{\Omega})^{l}$ for $u \in L^{r}(\tilde{\Omega})$. In the subsequent subsection 3.3 we address $(3.3)$ in $L^{2}(\tilde{\Omega})$. Superlinear convergence is investigated in the final subsection. In case $r>2$ a lifting step is introduced to compensate the fact that (3.3) is only available in $L^{2}(\tilde{\Omega})$.

Throughout this section it will be convenient to utilize the operator

$$
B u=G A^{-1} E_{\tilde{\Omega}} u,
$$

which satisfies $B \in \mathcal{L}\left(L^{r}(\tilde{\Omega}), \mathcal{C}(\bar{\Omega})^{l}\right)$ if (H2) and (H3) are satisfied. In particular $B^{*} \in \mathcal{L}\left(L^{s}(\Omega)^{l}, L^{r^{\prime}}(\tilde{\Omega})\right)$ for every $s \in(1, \infty)$. We shall require the following two additional hypotheses for some $\hat{r}>r$ :

$$
u_{d}, \bar{\varphi}, \underline{\varphi} \in L^{\hat{r}}(\tilde{\Omega}) \text {, and } u \mapsto A^{-*} J_{1}^{\prime}\left(A^{-1} E_{\tilde{\Omega}} u\right) \text { is continuously }
$$

Frechet differentiable from $L^{2}(\tilde{\Omega}) \rightarrow L^{\hat{r}}(\Omega)$, 
and

$$
B^{*} \in \mathcal{L}\left(L^{r}(\tilde{\Omega})^{l}, L^{\hat{r}}(\tilde{\Omega})\right) \quad \text { where } \frac{1}{\hat{r}}+\frac{1}{\hat{r}^{\prime}}=1 .
$$

We interpret the hypotheses (H5) and (H6) in view of the examples 1 and 2 in section 2 .

Example 1 (revisited). We have $G=\mathrm{id}$ and, hence, $B=A^{-1} E_{\tilde{\Omega}}$. Note that $A: W_{0}^{1, r^{\prime}}(\Omega) \rightarrow W^{-1, r^{\prime}}(\Omega)$ is a homeomorphism. Consequently, $A^{-*} \in \mathcal{L}\left(W^{-1, r}(\Omega)\right.$, $\left.W^{1, r}(\Omega)\right)$. For every $d$ there exists $\hat{r}>r$ such that $W_{0}^{1, r}(\Omega)$ embeds continuously into $L^{\hat{r}}(\Omega)$. Therefore $A^{-*} \in \mathcal{L}\left(L^{r}(\Omega), L^{\hat{r}}(\Omega)\right)$ and $B^{*} \in \mathcal{L}\left(L^{r}(\tilde{\Omega}), L^{\hat{r}}(\tilde{\Omega})\right)$. Hence, assumption (H6) is satisfied. The second part of hypothesis (H5) is fulfilled, e.g., for the tracking-type objective functional $J_{1}(y)=\frac{1}{2}\left|y-y_{d}\right|_{L^{2}(\Omega)}^{2}$ with $y_{d} \in L^{2}(\Omega)$ given.

Example 2 (revisited). Differently to example 1 we have $G=\nabla$ and, thus, $B=$ $\nabla A^{-1} E_{\tilde{\Omega}}$. Since $G^{*} \in \mathcal{L}\left(L^{r}(\Omega), W^{-1, r}(\Omega)\right)$ we have $A^{-*} G^{*} \in \mathcal{L}\left(L^{r}(\Omega), W^{1, r}(\Omega)\right)$. As in example 1 there exists for every $d$ some $\hat{r}>r$ such that $B^{*} \in E_{\tilde{\Omega}}^{*} A^{-*} G^{*} \in$ $\mathcal{L}\left(L^{r}(\Omega)^{l}, L^{\hat{r}}(\Omega)\right)$. For $J_{1}$ as in example 1 above (H5) is satisfied.

Next we note that $\bar{\mu}_{\gamma}$ and $\underline{\mu}_{\gamma}$ in $\left(\mathrm{OS}_{\gamma}\right)$ may be condensed into one multiplier $\mu_{\gamma}:=\bar{\mu}_{\gamma}-\underline{\mu}_{\gamma}$. Then the fourth and fifth equations of $\left(\mathrm{OS}_{\gamma}\right)$ are equivalent to

$$
\mu_{\gamma}=\left(\mu_{\gamma}+c\left(u_{\gamma}-\bar{\varphi}\right)\right)^{+}+\left(\mu_{\gamma}+c\left(u_{\gamma}-\underline{\varphi}\right)\right)^{-}
$$

for some $c>0$. Fixing $c=\alpha$ and using the third equation in $\left(\mathrm{OS}_{\gamma}\right)$ results in

$$
\alpha\left(u_{\gamma}-u_{d}\right)-E_{\tilde{\Omega}}^{*} p_{\gamma}+\left(E_{\tilde{\Omega}}^{*} p_{\gamma}+\alpha\left(u_{d}-\bar{\varphi}\right)\right)^{+}+\left(E_{\tilde{\Omega}}^{*} p_{\gamma}+\alpha\left(u_{d}-\underline{\varphi}\right)\right)^{-}=0
$$

Finally, using the state and the adjoint equation to express $y_{\gamma}$ and $p_{\gamma}$ in terms of $u_{\gamma}$, $\left(\mathrm{OS}_{\gamma}\right)$ turns out to be equivalent to

$$
F_{\gamma}\left(u_{\gamma}\right)=0, \quad F_{\gamma}: L^{r}(\tilde{\Omega}) \rightarrow L^{r}(\tilde{\Omega})
$$

with

$$
F_{\gamma}\left(u_{\gamma}\right):=\alpha\left(u_{\gamma}-u_{d}\right)-\hat{p}_{\gamma}+\left(\hat{p}_{\gamma}+\alpha\left(u_{d}-\bar{\varphi}\right)\right)^{+}+\left(\hat{p}_{\gamma}+\alpha\left(u_{d}-\underline{\varphi}\right)\right)^{-}
$$

and

$$
\hat{p}_{\gamma}:=p_{\gamma}\left(u_{\gamma}\right)=-\gamma B^{*}\left(\left|B u_{\gamma}\right|-\psi\right)^{+} q\left(B u_{\gamma}\right)-E_{\tilde{\Omega}}^{*} A^{-*} J_{1}^{\prime}\left(A^{-1} E_{\tilde{\Omega}} u_{\gamma}\right),
$$

where

$$
q(B u)(\mathrm{x})= \begin{cases}\left(\frac{B u}{|B u|}\right)(\mathrm{x}) & \text { if }|B u(\mathrm{x})|>0 \\ 0 & \text { otherwise. }\end{cases}
$$

We further set

$$
\mathfrak{p}_{\gamma}(u):=-\gamma B^{*}(|B u|-\psi)^{+} q(B u), \quad \text { where } \mathfrak{p}_{\gamma}: L^{r}(\tilde{\Omega}) \rightarrow L^{\hat{r}}(\tilde{\Omega}) .
$$

For the semismoothness of $F_{\gamma}$ we first study the Newton differentiability of $\mathfrak{p}_{\gamma}(\cdot)$. For its formulation we need

$$
G_{\max }(\omega)(\mathrm{x}):= \begin{cases}1 & \text { if } \omega(\mathrm{x})>0 \\ 0 & \text { if } \omega(\mathrm{x}) \leq 0\end{cases}
$$

Copyright $($ C by SIAM. Unauthorized reproduction of this article is prohibited. 
which was shown in [8] to serve as a generalized derivative for $\max (0, \cdot): L^{s_{1}}(\Omega) \rightarrow$ $L^{s_{2}}(\Omega)$ if $1 \leq s_{2}<s_{1} \leq \infty$. An analogous result holds true for $\min (0, \cdot)$. Further the norm-functional $|\cdot|: L^{s_{1}}(\Omega)^{l} \rightarrow L^{s_{2}}(\Omega)$, with $s_{1}, s_{2}$ as above, is Newton differentiable with generalized derivative $q(\cdot)$. This follows from Example 8.1 and Theorem 8.1 in [13]. There only the case $l=1$ is treated, but the result can be extended in a straightforward way to $l>1$.

We define

$$
Q(B v):=|B v|^{-1}\left(\mathrm{id}-|B v|^{-2}(B v)(B v)^{\top}\right) .
$$

Throughout this section, whenever we refer to (H3) it would actually suffice to have $G \in \mathcal{L}\left(W, \mathcal{C}(\bar{\Omega})^{l}\right)$.

Lemma 3.1. Assume that (H2), (H3), and (H6) hold true. Then the mapping $\mathfrak{p}_{\gamma}: L^{r}(\tilde{\Omega}) \rightarrow L^{\hat{r}}(\tilde{\Omega})$ is Newton differentiable in a neighborhood of every point $u \in$ $L^{r}(\tilde{\Omega})$ and a generalized derivative is given by

$$
G_{\mathfrak{p}_{\gamma}}(u)=-\gamma B^{*}\left[G_{\max }(|B u|-\psi) q(B u) q(B u)^{\top}+(|B u|-\psi)^{+} Q(B u)\right] B .
$$

Proof. By $(\mathrm{H} 6)$ there exists a constant $C_{1}(\gamma)$ such that

$$
\left\|\gamma B^{*}\right\|_{\mathcal{L}\left(L^{r}(\Omega), L^{\hat{r}}(\tilde{\Omega})\right)} \leq C_{1}(\gamma) .
$$

Let $u$ and $h \in L^{r}(\tilde{\Omega})$. Then we have by the definition of $\mathfrak{p}_{\gamma}$ in (3.7) and the expression for $G_{\mathfrak{p}_{\gamma}}$ in (3.8)

$$
\begin{aligned}
&\left|\mathfrak{p}_{\gamma}(u+h)-\mathfrak{p}_{\gamma}(u)-G_{\mathfrak{p}_{\gamma}}(u+h) h\right|_{L^{\hat{r}}(\tilde{\Omega})} \\
& \leq C_{1}(\gamma) \mid(|B(u+h)|-\psi)^{+} q(B u+h)-(|B u|-\psi)^{+} q(B u) \\
& \quad-\left[G_{\max }(|B(u+h)|-\psi) q(B(u+h)) q(B(u+h))^{\top}\right. \\
&\left.+(|B(u+h)|-\psi)^{+} Q(B(u+h))\right]\left.B h\right|_{L^{r}(\Omega)^{l}} \\
& \leq C_{1}(\gamma)\left|(|B(u+h)|-\psi)^{+}(q(B(u+h))-q(B u)-Q(B(u+h)) B h)\right|_{L^{r}(\Omega)^{l}} \\
&+C_{1}(\gamma) \mid\left((|B(u+h)|-\psi)^{+}-(|B u|-\psi)^{+}\right) q(B u) \\
& \quad-\left.\left[G_{\max }(|B(u+h)|-\psi) q(B(u)) q(B(u+h))^{\top}\right] B h\right|_{L^{r}(\Omega)^{l}} \\
&+C_{1}(\gamma) \mid G_{\max }(|B(u+h)|-\psi)(q(B u)-q(B(u+h))) q\left(\left.B(u+h)^{\top} B\right|_{L^{r}(\Omega)}\right. \\
&=I+I I+I I I .
\end{aligned}
$$

We now estimate separately the terms $I-I I I$. Let

$$
S=\left\{\mathrm{x}:|B u(\mathrm{x})| \leq \frac{\psi(\mathrm{x})}{2}\right\} .
$$

Then there exists $U(u) \subset L^{r}(\tilde{\Omega})$ and $\delta>0$ such that

$$
|B(u(\mathrm{x})+h(\mathrm{x}))| \leq \psi(\mathrm{x}), \quad \text { for all } \quad \mathrm{x} \in S, \quad u \in U(u), \quad|h|_{L^{r}(\tilde{\Omega})} \leq \delta,
$$

where we use that $B \in \mathcal{L}\left(L^{r}(\tilde{\Omega}), \mathcal{C}(\tilde{\Omega})^{l}\right)$ due to (H2) and (H3). Consequently

$$
I \leq C|q(B(u+h))-q(B u)-Q(B(u+h)) B h|_{\mathcal{C}(\overline{\Omega \backslash S})^{l}}
$$

Copyright $@$ by SIAM. Unauthorized reproduction of this article is prohibited. 
where $C=C(u, \delta)$. We check that $H: v \rightarrow \frac{v}{|v|}$ from $\mathcal{C}(\overline{\Omega \backslash S})^{l}$ to itself is uniformly Fréchet differentiable with Fréchet derivative

$$
H^{\prime}(v)=\frac{1}{|v|}\left(\mathrm{id}-\frac{v v^{\top}}{|v|^{2}}\right)
$$

provided that $v(\mathrm{x}) \neq 0$ for all $\mathrm{x} \in \overline{\Omega \backslash S}$. Moreover $v \rightarrow H^{\prime}(v)$ is locally Lipschitz continuous from $\mathcal{C}(\overline{\Omega \backslash S})^{l}$ to $\mathcal{C}(\overline{\Omega \backslash S})^{l \times l}$. Together with $B \in \mathcal{L}\left(L^{r}(\Omega), \mathcal{C}(\bar{\Omega})^{l}\right)$ this implies that

$$
I=\mathcal{O}\left(|h|_{L^{r}(\tilde{\Omega})}^{2}\right),
$$

where $\mathcal{O}$ is uniform with respect to $u \in U$. We turn to estimate $I I$ and consider $u \rightarrow(|B u|-\psi)^{+}$in the neighborhood $U$ of $u$. As noted above $G: v \rightarrow|v|$ is Newton differentiable from $L^{s_{1}}(\Omega)^{l}$ to $L^{s_{2}}(\Omega)$ if $1 \leq s_{2}<s_{1} \leq \infty$ at every $v \in L^{s_{1}}(\Omega)^{l}$, with a generalized derivative $\frac{v}{|v|}$, if $|v| \neq 0$. This, together with the chain rule for Newton differentiable maps composed with Frechet differentiable maps, see, e.g., [13], Lemma 8.1 or [14], and $B \in \mathcal{L}\left(L^{r}(\tilde{\Omega}), \mathcal{C}(\bar{\Omega})^{l}\right)$ (hence $B \in \mathcal{L}\left(L^{r}(\tilde{\Omega}), L^{r+2 \epsilon}(\Omega)^{l}\right)$ ) implies that $u \rightarrow|B u|$ is Newton differentiable from $L^{r}(\tilde{\Omega})$ to $L^{r+\varepsilon}(\Omega)$ for some $\varepsilon>0$, with a generalized derivative given by $q(u)$. Newton differentiability of this mapping also follows from [28], Theorem 5.2. The chain rule for two superimposed Newton differentiable maps given in Proposition A.1 implies then that $u \rightarrow(|B u|-\psi)^{+}$is Newton differentiable from $L^{r}(\tilde{\Omega})$ to $L^{r}(\Omega)$ and hence

$$
I I=\mathcal{O}\left(|h|_{L^{r}(\tilde{\Omega})}\right),
$$

with $\mathcal{O}$ uniform with respect to $u \in U$. It is straightforward to argue that

$$
I I I=\mathcal{O}\left(|h|_{L^{r}(\tilde{\Omega})}^{2}\right),
$$

with $\mathcal{O}$ uniform in $u \in U$. Combining (3.9)-(3.11) we have shown that

$$
\left|\mathfrak{p}_{\gamma}(u+h)-\mathfrak{p}_{\gamma}(u)-G_{\mathfrak{p}_{\gamma}}(u+h) h\right|_{L^{\hat{r}}(\tilde{\Omega})}=\mathcal{O}\left(|h|_{L^{r}(\tilde{\Omega})}\right),
$$

as $\left.|h|_{L^{r}(\tilde{\Omega})}\right) \rightarrow 0$, with $\mathcal{O}$ uniform in $u \in U$. Hence, $\mathfrak{p}_{\gamma}$ is Newton differentiable in the neighborhood $U$ of $u$.

Newton differentiability of $F_{\gamma}$ is established next.

Theorem 3.1. Let (H2), (H3), (H5), and (H6) hold true. Then $F_{\gamma}: L^{r}(\tilde{\Omega}) \rightarrow$ $L^{r}(\tilde{\Omega})$ is Newton differentiable in a neighborhood of every $u \in L^{r}(\tilde{\Omega})$.

Proof. We consider the various constituents of $F_{\gamma}$ separately. In terms of

$$
\hat{p}_{\gamma}(u):=\mathfrak{p}_{\gamma}(u)-E_{\tilde{\Omega}}^{*} A^{-*} J_{1}^{\prime}\left(A^{-1} E_{\tilde{\Omega}} u\right),
$$

we have by (3.6)

$$
F_{\gamma}(u)=\alpha\left(u-u_{d}\right)-\hat{p}_{\gamma}(u)+\left(\hat{p}_{\gamma}(u)+\alpha\left(u_{d}-\bar{\varphi}\right)\right)^{+}+\left(\hat{p}_{\gamma}(u)+\alpha\left(u_{d}-\underline{\varphi}\right)\right)^{-} .
$$

Lemma 3.1 and (H5) for $J_{1}$ yield the Newton differentiability of

$$
u \mapsto \alpha\left(u-u_{d}\right)-\hat{p}_{\gamma}(u) \text { from } L^{r}(\tilde{\Omega}) \text { to } L^{r}(\tilde{\Omega}),
$$

in a neighborhood $U(u)$ of $u$. 
We further have by Lemma 3.1 that

$$
\hat{p}_{\gamma}(\cdot)+\alpha\left(u_{d}-\bar{\varphi}\right) \text { and } \hat{p}_{\gamma}(\cdot)+\alpha\left(u_{d}-\underline{\varphi}\right)
$$

are locally Lipschitz continuous and Newton differentiable from $L^{r}(\tilde{\Omega})$ to $L^{\hat{r}}(\tilde{\Omega})$, respectively. Then the results of Appendix A yield the Newton differentiability of

$$
\left(\hat{p}_{\gamma}(\cdot)+\alpha\left(u_{d}-\bar{\varphi}\right)\right)^{+}+\left(\hat{p}_{\gamma}(\cdot)+\alpha\left(u_{d}-\underline{\varphi}\right)\right)^{-}
$$

from $L^{r}(\tilde{\Omega})$ to $L^{r}(\tilde{\Omega})$ in a, possibly smaller neighborhood $U(u)$ of $u$. Combining these results proves the assertion.

The structure of a particular generalized derivative associated with $F_{\gamma}$ immediately follows from a combination of the previous results.

COROLlaRY 3.1. Let the assumptions of Theorem 3.1 hold. Then a particular generalized derivative of $F_{\gamma}$ at $u \in L^{r}(\tilde{\Omega})$ is given by

$$
\begin{gathered}
G_{F_{\gamma}}(u)=\alpha \mathrm{id}-G_{\hat{p}_{\gamma}}(u)+G_{\max }\left(\hat{p}_{\gamma}(u)+\alpha\left(u_{d}-\bar{\varphi}\right)\right) G_{\hat{p}_{\gamma}}(u) \\
+G_{\min }\left(\hat{p}_{\gamma}(u)+\alpha\left(u_{d}-\underline{\varphi}\right)\right) G_{\hat{p}_{\gamma}}(u)
\end{gathered}
$$

with

$$
G_{\hat{p}_{\gamma}}(u)=G_{\mathfrak{p}_{\gamma}}(u)-E_{\tilde{\Omega}}^{*} A^{-*} J_{1}^{\prime \prime}\left(A^{-1} E_{\tilde{\Omega}} u\right) A^{-1} E_{\tilde{\Omega}} .
$$

3.2. Uniform boundedness of the inverse of the generalized derivative in $\boldsymbol{L}^{\mathbf{2}}(\tilde{\boldsymbol{\Omega}})$. Next we study $G_{F_{\gamma}}$ in more detail. For a well-defined semismooth Newton step, we need its nonsingularity on a particular subspace. Given an approximation $u^{k}$ of $u_{\gamma}$, in our context the semismooth Newton update step is defined as

$$
G_{F_{\gamma}}\left(u^{k}\right) \delta_{u}^{k}=-F_{\gamma}\left(u^{k}\right)
$$

with $\delta_{u}^{k}=u^{k+1}-u^{k}$; compare (3.2) with $x=u$ and $F=F_{\gamma}$.

For our subsequent investigation we define the active and inactive sets

$$
\begin{aligned}
\overline{\mathcal{A}}^{k} & :=\left\{\mathrm{x} \in \tilde{\Omega}:\left(\hat{p}_{\gamma}\left(u^{k}\right)+\alpha\left(u_{d}-\bar{\varphi}\right)\right)(\mathrm{x})>0\right\}, \\
\underline{\mathcal{A}}^{k} & :=\left\{\mathrm{x} \in \tilde{\Omega}:\left(\hat{p}_{\gamma}\left(u^{k}\right)+\alpha\left(u_{d}-\underline{\varphi}\right)\right)(\mathrm{x})<0\right\}, \\
\mathcal{A}^{k} & :=\overline{\mathcal{A}}^{k} \cup \underline{\mathcal{A}}^{k}, \\
\mathcal{I}^{k} & :=\tilde{\Omega} \backslash \mathcal{A}^{k} .
\end{aligned}
$$

Further we introduce $\chi_{\mathcal{I}^{k}}$, the characteristic function of the inactive set $\mathcal{I}^{k}$, and the extension-by-zero operators $E_{\overline{\mathcal{A}}^{k}}, E_{\underline{\mathcal{A}}^{k}}, E_{\mathcal{A}^{k}}$, and $E_{\mathcal{I}^{k}}$ with the properties $E_{\mathcal{A}^{k}} \chi_{\mathcal{I}^{k}}=0$ and $E_{\mathcal{I}^{k}} \chi_{\mathcal{I}^{k}}=\chi_{\mathcal{I}^{k}}$.

Corollary 3.1 and the structure of $G_{\max }$ and $G_{\min }$, respectively, yield that

$$
G_{F_{\gamma}}\left(u^{k}\right)=\alpha \operatorname{id}-\chi_{\mathcal{I}^{k}} G_{\hat{p}_{\gamma}}\left(u^{k}\right) .
$$

Hence, from the restriction of (3.12) to $\overline{\mathcal{A}}^{k}$ we find that

$$
\delta_{u \mid \overline{\mathcal{A}}^{k}}^{k}=E_{\overline{\mathcal{A}}^{k}}^{*} \delta_{u}^{k}=E_{\overline{\mathcal{A}}^{k}}^{*}\left(\bar{\varphi}-u^{k}\right)=\bar{\varphi}_{\mid \overline{\mathcal{A}}^{k}}-u_{\mid \overline{\mathcal{A}}^{k}}^{k}
$$

and similarly

$$
\delta_{u \mid \underline{\mathcal{A}}^{k}}^{k}=E_{\underline{\mathcal{A}}^{k}}^{*} \delta_{u}^{k}=E_{\underline{\mathcal{A}}^{k}}^{*}\left(\underline{\varphi}-u^{k}\right)=\underline{\varphi} \mid \underline{\mathcal{A}}^{k}-u_{\left.\right|_{\underline{\mathcal{A}}^{k}} ^{k}}^{k}
$$

Copyright $@$ by SIAM. Unauthorized reproduction of this article is prohibited. 
Hence, $\delta_{u \mid \mathcal{A}^{k}}^{k}$ is obtained by a simple assignment of data according to the previous iterate only. Therefore, it remains to study (3.12) on the inactive set, i.e.

$$
E_{\mathcal{I}^{k}}^{*} G_{F_{\gamma}}\left(u^{k}\right) E_{\mathcal{I}^{k}} \delta_{u}^{\mathcal{I}^{k}}=-E_{\mathcal{I}^{k}}^{*}\left(F_{\gamma}\left(u^{k}\right)+G_{F_{\gamma}}\left(u^{k}\right) E_{\mathcal{A}^{k}} \delta_{u \mid \mathcal{A}^{k}}^{k}\right)
$$

as an equation in $L^{2}\left(\mathcal{I}^{k}\right)$.

Lemma 3.2. Let (H2), (H3), (H5), and (H6) hold and $\mathcal{I} \subset \Omega$. Then the inverse to the operator

$$
E_{\mathcal{I}}^{*} G_{F_{\gamma}}(u) E_{\mathcal{I}}: L^{2}(\mathcal{I}) \rightarrow L^{2}(\mathcal{I}),
$$

with $G_{F_{\gamma}}(u)=\alpha \operatorname{id}-\chi_{\mathcal{I}} G_{\hat{p}_{\gamma}}(u)$, exists and is bounded by $\frac{1}{\alpha}$ regardless of $u \in L^{r}(\tilde{\Omega})$ as long as meas $(\mathcal{I})>0$.

Proof. Note that we have

$$
E_{\mathcal{I}}^{*} G_{F_{\gamma}}(u) E_{\mathcal{I}}=\alpha \operatorname{id}_{\mid \mathcal{I}}+\gamma E_{\mathcal{I}}^{*} B^{*} T(u) B E_{\mathcal{I}}+E_{\mathcal{I}}^{*} E_{\tilde{\Omega}}^{*} A^{-*} J_{1}^{\prime \prime}\left(A^{-1} E_{\tilde{\Omega}} u\right) A^{-1} E_{\tilde{\Omega}} E_{\mathcal{I}}
$$

with

$$
T(u)=G_{\max }(|B u|-\psi) q(B u) q(B u)^{\top}+(|B u|-\psi)^{+} Q(B u) .
$$

From $B \in \mathcal{L}\left(L^{\hat{r}^{\prime}}(\tilde{\Omega}), L^{\hat{r}^{\prime}}(\Omega)\right) \cap \mathcal{L}\left(L^{r}(\tilde{\Omega}), L^{r}(\Omega)\right)$, by (H2), (H3), and (H6), we conclude by interpolation that $B \in \mathcal{L}\left(L^{2}(\tilde{\Omega}), L^{2}(\Omega)\right)$. Moreover, $T(u) \in \mathcal{L}\left(L^{2}(\Omega)\right)$. Therefore

$$
\gamma E_{\mathcal{I}}^{*} B^{*} T(u) B E_{\mathcal{I}} \in L^{2}(\tilde{\Omega}) \text { and } E_{\mathcal{I}}^{*} E_{\tilde{\Omega}}^{*} A^{-*} J_{1}^{\prime \prime}\left(A^{-1} E_{\tilde{\Omega}} u\right) A^{-1} E_{\tilde{\Omega}} E_{\mathcal{I}} \in L^{2}(\tilde{\Omega}),
$$

where we also use (H5). In conclusion, the operator $E_{\mathcal{I}}^{*} G_{F_{\gamma}}(u) E_{\mathcal{I}}$ is an element of $\mathcal{L}\left(L^{2}(\mathcal{I})\right)$. From the convexity of $J$ we infer for arbitrary $z \in L^{r}(\mathcal{I})$ that

$$
\left(\left(\alpha \operatorname{id}_{\mid \mathcal{I}}+E_{\mathcal{I}}^{*} E_{\tilde{\Omega}}^{*} A^{-*} J_{1}^{\prime \prime}\left(A^{-1} E_{\tilde{\Omega}} u\right) A^{-1} E_{\tilde{\Omega}} E_{\mathcal{I}}\right) z, z\right)_{L^{2}(\mathcal{I})} \geq \alpha\|z\|_{L^{2}(\mathcal{I})}^{2} .
$$

Turning to $\gamma E_{\mathcal{I}}^{*} B^{*} T(u) B E_{\mathcal{I}}$ we observe that $T(u)=0$ in $\{|B u|-\psi \leq 0\}$ and $0<\psi /|B u|-1<1$ in $\{|B u|-\psi>0\}$. Hence,

$$
(T(u) w, w)_{L^{2}(\tilde{\Omega})}=\int_{\{|B u|-\psi>0\}}\left(1-\frac{\psi}{|B u|}\right)|w|^{2} \geq 0
$$

for all $w \in L^{2}(\tilde{\Omega})$. From this and (3.20) we conclude that the inverse to $E_{\mathcal{I}}^{*} G_{F_{\gamma}}(u) E_{\mathcal{I}}$ : $L^{2}(\mathcal{I}) \rightarrow L^{2}(\mathcal{I})$ is bounded by $\frac{1}{\alpha}$.

Proposition 3.1. If $(\mathrm{H} 2)$, ( $\mathrm{H} 3)$, (H5), and $(\mathrm{H} 6)$ hold, then the semismooth Newton update step $(3.12)$ is well-defined and $\delta_{u}^{k} \in L^{r}(\tilde{\Omega})$.

Proof. Well-posedness of the Newton step with $\delta_{u}^{k} \in L^{2}(\tilde{\Omega})$ follows immediately from (3.17), (3.18), and Lemma 3.2. Note that whenever $\mathcal{I}^{k}=\emptyset$, then $\delta_{u}^{k}$ is fully determined by (3.17) and (3.18). An inspection of (3.17), (3.18), and (3.19), using (H5) and the structure of $E_{\mathcal{I}}^{*} G_{F_{\gamma}}(u) E_{\mathcal{I}}$, moreover, shows that $\delta_{u}^{k} \in L^{r}(\tilde{\Omega})$.

From Lemma 3.2 and the proof of Proposition 3.1 we conclude that $E_{\mathcal{I}}^{*} G_{F_{\gamma}}$ $(u) E_{\mathcal{I}} v=f$ is solvable in $L^{r}(\tilde{\Omega})$ if $f \in L^{r}(\tilde{\Omega})$. It is not clear, however, whether $\left(E_{\mathcal{I}}^{*} G_{F_{\gamma}}(u) E_{\mathcal{I}}\right)^{-1}$ is bounded as an operator in $\mathcal{L}\left(L^{r}(\tilde{\Omega})\right)$ uniformly with respect to $u$.

We are now prepared to consider (3.3) for $G_{F_{\gamma}}$ specified in Corollary 3.1.

Proposition 3.2. Let (H2), (H3), (H5), and (H6) hold. Then for each $\hat{u} \in$ $L^{r}(\tilde{\Omega})$ there exists a neighborhood $U(\hat{u}) \subset L^{r}(\tilde{\Omega})$ and a constant $K$ such that

$$
\left\|G_{F_{\gamma}}(u)^{-1}\right\|_{\mathcal{L}\left(L^{2}(\hat{\Omega})\right)} \leq K \text { for all } u \in U(\hat{u}) .
$$

Copyright $@$ by SIAM. Unauthorized reproduction of this article is prohibited. 
Proof. Let $\mathcal{A}$ and $\mathcal{I}$ denote disjoint subsets of $\tilde{\Omega}$ such that $\mathcal{A} \cup \mathcal{I}=\tilde{\Omega}$. Then observe that every $v \in L^{2}(\tilde{\Omega})$ can be uniquely decomposed in two components $\left(E_{\mathcal{I}}^{*} v, E_{\mathcal{A}}^{*} v\right)$. For $g \in L^{2}(\tilde{\Omega})$ the equation

$$
G_{F_{\gamma}}(u) v=g
$$

is equivalent to

$$
\left\{\begin{array}{l}
E_{\mathcal{A}}^{*} v=E_{\mathcal{A}}^{*} g, \\
\left(E_{\mathcal{I}}^{*} G_{F_{\gamma}}(u) E_{\mathcal{I}}\right) E_{\mathcal{I}}^{*} v=E_{\mathcal{I}}^{*} g-E_{\mathcal{I}}^{*} G_{F_{\gamma}}(u) E_{\mathcal{A}} E_{\mathcal{A}}^{*} g .
\end{array}\right.
$$

In the proof of Lemma 3.2 we argued that

$$
G_{F_{\gamma}}(u) \in \mathcal{L}\left(L^{2}(\tilde{\Omega})\right) \text {, for each } u \in L^{2}(\tilde{\Omega}) .
$$

Slightly generalizing this argument shows that for each $\hat{u} \in L^{2}(\tilde{\Omega})$ there exists a neighborhood $U(\hat{u})$ and $C_{\hat{u}}$ such that

$$
\left\|G_{F_{\gamma}}(u)\right\|_{\mathcal{L}\left(L^{2}(\tilde{\Omega})\right)} \leq C_{\hat{u}} \text { for all } u \in U(\hat{u}) .
$$

From (3.22) and Lemma 3.2 it follows that (3.21) holds with $K=1+\frac{1}{\alpha}\left(1+C_{\hat{u}}\right)$.

3.3. Local q-superlinear convergence of the semismooth Newton iteration without and with a lifting step. For $r=2$ we can deduce the following result from the discussion at the beginning of section 3, Lemma 3.1, and Proposition 3.2.

Theorem 3.2. If (H2), (H3), (H5), and (H6) hold, then the semismooth Newton iteration (3.2) applied to $F_{\gamma}$ given in (3.6) with generalized derivative $G_{F_{\gamma}}$, given in Corollary 3.1 , is locally q-superlinearly convergent in $L^{2}(\tilde{\Omega})$.

In case $r>2$ the semismooth Newton algorithm is supplemented by a lifting step.

Algorithm 2 (semismooth Newton method with lifting).

(i) Choose $u^{0} \in L^{r}(\tilde{\Omega})$.

(ii) Solve for $\tilde{u}^{k+1} \in L^{r}(\tilde{\Omega})$ :

$$
G_{F_{\gamma}}\left(u^{k}\right)\left(\tilde{u}^{k+1}-u^{k}\right)=-F_{\gamma}\left(u^{k}\right) .
$$

(iii) Perform a lifting step:

$$
u^{k+1}=\frac{1}{\alpha}\left(u_{d}+p_{\gamma}-\left(p_{\gamma}+\alpha\left(u_{d}-\bar{\varphi}\right)\right)^{+}-\left(p_{\gamma}+\alpha\left(u_{d}-\underline{\varphi}\right)\right)^{-}\right),
$$

where $p_{\gamma}=p_{\gamma}\left(\tilde{u}^{k+1}\right)$.

The case with $r>2$ is addressed next.

TheOrem 3.3. If (H2), (H3), (H5), and (H6) hold, then the semismooth Newton method with lifting step is locally q-superlinearly convergent in $L^{r}(\tilde{\Omega})$.

Proof. Let $U\left(u_{\gamma}\right)$ denote the neighborhood of $u_{\gamma}$ according to Theorem 3.1. Proposition 3.2 implies the existence of a constant $M$ and $\bar{\rho}>0$ such that

$$
\left\|G_{F_{\gamma}}^{-1}(u)\right\|_{\mathcal{L}\left(L^{2}(\tilde{\Omega})\right)} \leq M
$$

for all $u \in B_{r}\left(u_{\gamma}, \rho\right)$. Here $B_{r}\left(u_{\gamma}, \rho\right)$ denotes the open ball with radius $\rho$ and center $u_{\gamma}$ in $L^{r}(\tilde{\Omega})$, with $\rho$ sufficiently small such that $B_{r}\left(u_{\gamma}, \rho\right) \subset U\left(u_{\gamma}\right)$. We recall the definition of $p_{\gamma}(u)$ :

$$
p_{\gamma}(u)=-\gamma B^{*}(|B u|-\psi)^{+} q(B u)-E_{\tilde{\Omega}}^{*} A^{-*} J_{1}^{\prime}\left(A^{-1} E_{\tilde{\Omega}} u\right) .
$$

Copyright $@$ by SIAM. Unauthorized reproduction of this article is prohibited. 
A computation shows that $v \rightarrow(|v|-\psi)^{+} q(v)$ is globally Lipschitz continuous from $L^{r}(\tilde{\Omega})^{l}$ to itself with Lipschitz constant 3. Since $B \in \mathcal{L}\left(L^{r}(\tilde{\Omega}), L^{\infty}(\Omega)\right)$ by (H3) and $B \in \mathcal{L}\left(L^{\hat{r}^{\prime}}(\tilde{\Omega}), L^{r^{\prime}}(\Omega)\right)$ by (H6), the Marcinkiewicz interpolation theorem then implies that $B \in \mathcal{L}\left(L^{2}(\tilde{\Omega}), L^{r}(\Omega)\right)$. Moreover $B^{*} \in \mathcal{L}\left(L^{r}(\tilde{\Omega}), L^{r}(\Omega)\right)$ again as a consequence of (H6), and hence the first summand in (3.23) is globally Lipschitz continuous from $L^{2}(\tilde{\Omega})$ to $L^{r}(\Omega)$. This together with (H5) shows that $p_{\gamma}(u)$ is locally Lipschitz continuous from $L^{2}(\tilde{\Omega})$ to $L^{r}(\Omega)$. Let $L$ denote the Lipschitz constant of $p_{\gamma}(\cdot)$ in $B_{2}\left(u_{\gamma}, \bar{\rho} M\right) \subset L^{2}(\tilde{\Omega})$. Without loss of generality we assume that $\alpha<L M$.

With $L, M$, and $\bar{\rho}$ specified, the lifting property of $F_{\gamma}$ implies the existence of a constant $0<\rho<\bar{\rho}$ such that

$$
\left|F_{\gamma}\left(u_{\gamma}+h\right)-F_{\gamma}\left(u_{\gamma}\right)-G_{F_{\gamma}}\left(u_{\gamma}+h\right) h\right|_{L^{r}(\tilde{\Omega})} \leq \frac{\alpha}{3 L M|\Omega|^{\frac{r-2}{2}}}|h|_{L^{r}(\tilde{\Omega})}
$$

for all $|h|_{L^{r}(\tilde{\Omega})}<\rho$. Let $u_{0}$ be such that $u_{0} \in B_{2}\left(u_{\gamma}, \bar{\rho}\right) \cap B_{r}\left(u_{\gamma}, \bar{\rho}\right)$, and proceeding by induction, assume that $u_{k} \in B_{2}\left(u_{\gamma}, \bar{\rho}\right) \cap B_{r}\left(u_{\gamma}, \bar{\rho}\right)$. Then

$$
\begin{aligned}
\left|\tilde{u}^{k+1}-u_{\gamma}\right|_{L^{2}(\tilde{\Omega})} \leq & \left\|G_{F_{\gamma}}\left(u^{k}\right)^{-1}\right\|_{\mathcal{L}\left(L^{2}\right)}|\Omega|^{\frac{r-2}{r}} \\
& \cdot\left|F_{\gamma}\left(u^{k}\right)-F\left(u_{\gamma}\right)-G_{F_{\gamma}}\left(u^{k}\right)\left(u^{k}-u_{\gamma}\right)\right|_{L^{r}(\tilde{\Omega})} \\
\leq & \frac{\alpha}{3 L M}\left|u^{k}-u_{\gamma}\right|_{L^{r}(\tilde{\Omega})}<\left|u^{k}-u_{\gamma}\right|_{L^{r}(\tilde{\Omega})},
\end{aligned}
$$

and, in particular, $\tilde{u}_{k+1} \in B_{2}\left(u_{\gamma}, \bar{\rho}\right)$. We further investigate the implications of the lifting step:

$$
\begin{aligned}
u^{k+1}-u_{\gamma}=\frac{1}{\alpha}( & p_{\gamma}\left(\tilde{u}^{k+1}\right)-p_{\gamma}\left(u_{\gamma}\right)-\left(p_{\gamma}\left(\tilde{u}^{k+1}\right)+\alpha\left(u_{d}-\bar{\varphi}\right)\right)^{+} \\
& +\left(p_{\gamma}\left(u_{\gamma}\right)+\alpha\left(u_{d}-\bar{\varphi}\right)\right)^{+}-\left(p_{\gamma}\left(\tilde{u}^{k+1}\right)+\alpha\left(u_{d}-\underline{\varphi}\right)\right)^{-} \\
& \left.+\left(p_{\gamma}\left(u_{\gamma}\right)+\alpha\left(u_{d}-\underline{\varphi}\right)\right)^{+}\right)
\end{aligned}
$$

which implies that

$$
\left|u^{k+1}-u_{\gamma}\right|_{L^{r}(\tilde{\Omega})} \leq \frac{3}{\alpha}\left|p_{\gamma}\left(\tilde{u}^{k+1}\right)-p_{\gamma}\left(u_{\gamma}\right)\right|_{L^{r}(\Omega)} \leq \frac{3 L}{\alpha}\left|\tilde{u}^{k+1}-u_{\gamma}\right|_{L^{2}(\tilde{\Omega})} .
$$

Combining (3.24) and (3.25) implies that $u^{k+1} \in B_{r}\left(u_{\gamma}, \bar{\rho}\right)$. Thus the iteration is well-defined. Moreover we find

$$
\frac{\left|u^{k+1}-u_{\gamma}\right|_{L^{r}(\tilde{\Omega})}}{\left|u^{k}-u_{\gamma}\right|_{L^{r}(\tilde{\Omega})}} \leq \frac{\frac{3 L}{\alpha} M|\Omega|^{\frac{r-2}{r}}\left|F_{\gamma}\left(u^{k}\right)-F_{\gamma}\left(u^{\gamma}\right)-G_{F_{\gamma}}\left(u^{k}\right)\left(u^{k}-u_{\gamma}\right)\right|_{L^{r}(\tilde{\Omega})}}{\left|u^{k}-u_{\gamma}\right|_{L^{r}(\tilde{\Omega})}},
$$

which by (3.1) implies $q$-superlinear convergence.

Remark 3.1. If we had a uniform estimate on $\left\|G_{F_{\gamma}}\left(u^{k}\right)^{-1}\right\|_{\mathcal{L}\left(L^{r}(\tilde{\Omega})\right)}$, then the lifting step could be avoided. In fact, note that we are not using the full power of the semismooth estimate (3.1) in (3.24), since we overestimate the $L^{2}$-norm by the $L^{r}$-norm.

We note, however, that for each fixed $u \in L^{r}(\tilde{\Omega})$ the operator $G_{F_{\gamma}}(u)$ is continuously invertible from $L^{r}(\tilde{\Omega})$ to itself; see Proposition 3.1. Thus, if $u_{k} \mapsto G_{F_{\gamma}}\left(u_{k}\right)$ is continuous for all sufficiently large $k$, then the desired uniform estimate $G_{F_{\gamma}}\left(u_{k}\right)^{-1} \in$ 
$\mathcal{L}\left(L^{r}(\tilde{\Omega})\right)$ holds. This continuity cannot be expected in general since $G_{F_{\gamma}}(u)$ contains the operator

$$
T(u)=G_{\max }(|B u|-\psi) q(B u) q(B u)^{\top}+(|B u|-\psi)^{+} Q(B u) ;
$$

see Lemma 3.2. If, however, the measure of the set $\left\{\mathrm{x}:\left(\left|B u^{k}\right|-\psi\right)(\mathrm{x})>0\right\}$, and similarly for the max-term changes continuously with $k$, then the uniform bound on the inverse holds and the lifting step can be avoided. In the numerical examples given in the following section this behavior could be observed.

4. Numerics. Finally, we report on our numerical experience with Algorithm 1. In our tests we are interested in solving $\left(\mathrm{P}_{\gamma}\right)$ with large $\gamma$, i.e., we aim at a rather accurate approximation of the solution of $(\mathrm{P})$. Algorithmically this is achieved by preselecting a sequence $\gamma_{\ell}=10^{\ell}, \ell=0, \ldots, 8$, of $\gamma$-values and solving $\left(\mathrm{P}_{\gamma}\right)$ for $\gamma_{\ell}$ with the solution of the problem corresponding to $\gamma_{\ell-1}$ as the initial guess. For $\ell=0$ we use $u^{0} \equiv 0$ as the initial guess. Such a continuation strategy with respect to $\gamma$ is well-suited for producing initial guesses for the subsequent problem $\left(\mathrm{P}_{\gamma}\right)$ which satisfy the locality assumption of Theorem 3.2, and it usually results in a small number of semismooth Newton iterations until successful termination. We point out that more sophisticated and automatic selection rules for $\left(\gamma_{\ell}\right)$ may be used. For instance, one may adapt the technique of [9] for zero-order state constraints without additional constraints on the control.

In the numerical tests we throughout consider $A=-\Delta, \tilde{\Omega}=\Omega=(0,1)^{2}$, and $J_{1}(y)=\left\|y-y_{d}\right\|_{L^{2}(\Omega)}^{2}$. Here we discuss results for the following two problems.

Problem 1. The setting for this problem corresponds to Example 1. In this case we have zero-order state constraints, i.e., $G=\mathrm{id}$, with $\psi\left(x_{1}, x_{2}\right)=5 E-3(1+0.25 \mid 0.5-$ $\left.x_{1} \mid\right)$. The lower and upper bounds for the control are $\underline{\varphi} \equiv 0$ and $\bar{\varphi}\left(x_{1}, x_{2}\right)=0.1+$ $\left|\cos \left(2 \pi x_{1}\right)\right|$, respectively. Moreover we set $u_{d} \equiv 0, y_{d}\left(x_{1}, x_{2}\right)=\sin \left(2 \pi x_{1}\right) \exp \left(2 x_{2}\right) / 6$, and $\alpha=1 E-2$.

PROBLEM 2. The second example corresponds to first-order state constraints with $G=\nabla$ and $\psi \equiv 0.1$. The pointwise bounds on the control are

$$
\underline{\varphi}\left(x_{1}, x_{2}\right)= \begin{cases}-0.5-\left|x_{1}-0.5\right|-\left|x_{2}-0.5\right| & \text { if } x_{1}>0.5 \\ 0 & \text { if } x_{1} \leq 0.5\end{cases}
$$

and $\bar{\varphi}\left(x_{1}, x_{2}\right)=0.1+\left|\cos \left(2 \pi x_{1}\right)\right|$. The desired control $u_{d}$, the desired state $y_{d}$, and $\alpha$ are as in Problem 1.

For the discretization of the respective problem we choose a regular mesh with mesh size $h$. The discretization of $A$ is based on the standard five-point stencil and the one of $G$ in Problem 2 uses symmetric differences. For each $\gamma$-value the algorithm is stopped as soon as $\left\|A_{h} p_{h}+\gamma G_{h}^{\top}\left(\left|G_{h} y_{h}\right|-\psi_{h}\right)^{+}+J_{1 h}^{\prime}\left(y_{h}\right)\right\|_{-1}$ and $\left\|\mu_{h}-\left(\mu_{h}+\alpha\left(u_{d, h}-b_{h}\right)^{+}\right)-\left(\mu_{h}+\alpha\left(u_{d, h}-a_{h}\right)^{-}\right)\right\|_{2}$ drop below tol=1E-8. Here we use $\|w\|_{-1}=\left\|A_{h}^{-1} w\right\|_{2}$, and the subscript $h$ refers to discretized quantities. Before we commence with reporting on our numerical results, we briefly address step (ii) of Algorithm 2. In our tests, the solution of the linear system is achieved by sparse (Cholesky) factorization techniques. Alternatively, one may rely on iterative solvers (such as preconditioned conjugate gradient methods) for the state and the adjoint equation, respectively, as well as for the linear system in step (ii) of Algorithm 2.

In Figure 4.1 we display the state, control, and multiplier $\mu_{h}$ upon termination of Algorithm 1 when solving the discrete version of Problem 1 for $\gamma=1 \mathrm{E} 8$ and $h=1 / 256$. The active and inactive set structure with respect to the pointwise 

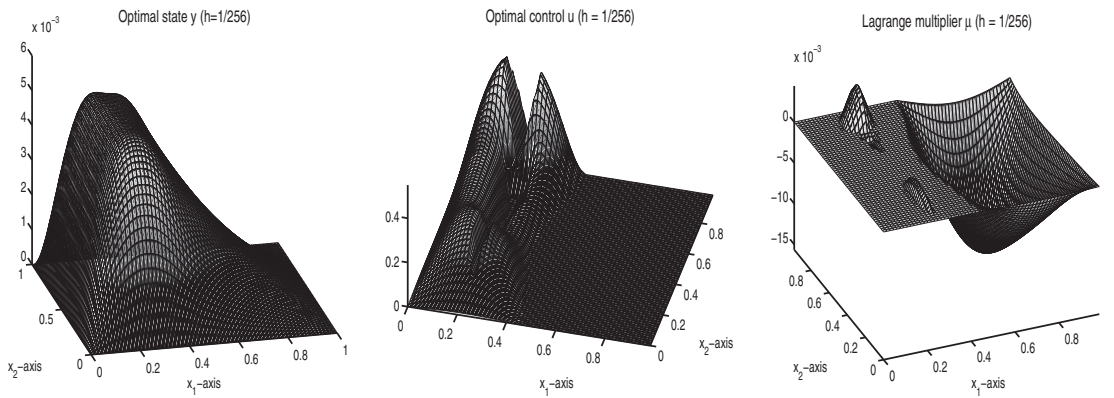

FIG. 4.1. Problem $1(\gamma=1 E 8, h=1 / 256)$. State $y_{h}$, control $u_{h}$, and multiplier $\mu_{h}$ upon termination of Algorithm 1.
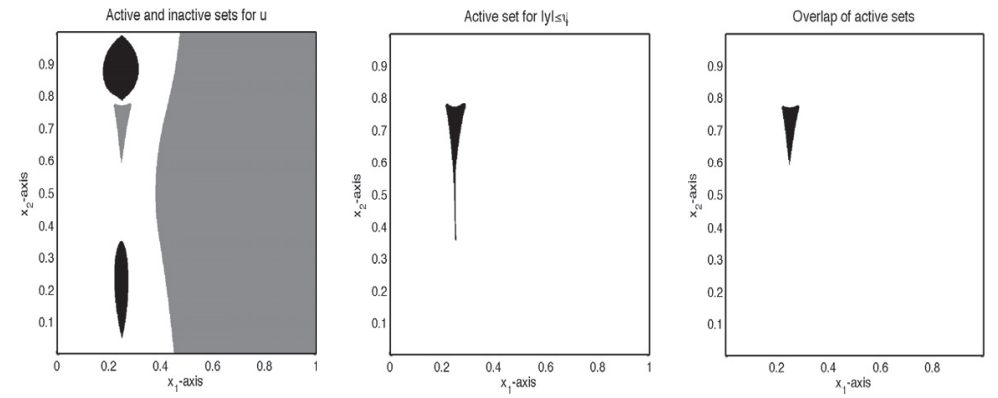

FIG. 4.2. Problem $1(\gamma=1 E 8, h=1 / 256)$. Inactive set (white), active set for the lower bound (gray), and for the upper bound (black) in the left plot. Approximation of the active set for the zero-order state constraint (black) in the middle plot. Overlap of active regions for control and state constraints in the right plot.

constraints on $u_{h}$ can be seen in the left plot of Figure 4.2. Here, the white region corresponds to the inactive set, the gray region represents the active set for the lower bound, and the black set is the active set with respect to the upper bound. The graph in the middle shows the approximation of the active set for the zero-order state constraint. On the right we show the overlapping region where the pointwise state constraint and one of the bounds on the control are active simultaneously. In Table 4.1 we display the iteration numbers upon successful termination of Algorithm 1 for various mesh sizes and for each $\gamma$-value of our preselected sequence. We recall that these figures are based on our $\gamma$-continuation strategy.

Upon studying the above results for Problem 1, we first note that Algorithm 1 with $\gamma$-continuation exhibits a mesh independent behavior. This can be seen from the stable iteration counts along the columns of Table 4.1. Moreover, for fixed $h$ the number of iterations until termination is rather stable as well. This is due to the excellent initial guesses produced by our $\gamma$-continuation technique. In our tests we also found that Algorithm 1 without the $\gamma$-continuation for solving $\left(\mathrm{P}_{\gamma}\right)$ for large $\gamma$ and with initial choice $u_{h}^{0}=0$ may fail to converge. Concerning the test example under investigation, we note that the overlap of the active sets for the state and the controls is rather special. In this case, the bound on the state and the control satisfy the state equation in the region of overlapping active sets.

Next we report on our findings for Problem 2. In Figure 4.3 we show the state, control, and multiplier $\mu_{h}$ upon termination for $\gamma=1 \mathrm{E} 8$ and $h=1 / 256$. Figure 4.4 shows the active and inactive sets for the constraints on the control in the left plot 
TABLE 4.1

Problem 1. Number of iterations for various mesh sizes and $\gamma$-values.

\begin{tabular}{|c||c|c|c|c|c|c|c|c|c|}
\hline \multicolumn{10}{|c|}{ Iterations } \\
\hline$h / \gamma$ & $1 \mathrm{E} 0$ & $1 \mathrm{E} 1$ & $1 \mathrm{E} 2$ & $1 \mathrm{E} 3$ & $1 \mathrm{E} 4$ & $1 \mathrm{E} 5$ & $1 \mathrm{E} 6$ & $1 \mathrm{E} 7$ & $1 \mathrm{E} 8$ \\
\hline \hline$\frac{1}{64}$ & 3 & 3 & 4 & 5 & 5 & 5 & 4 & 4 & 2 \\
\hline$\frac{1}{128}$ & 3 & 3 & 4 & 6 & 5 & 5 & 5 & 4 & 4 \\
\hline$\frac{1}{256}$ & 3 & 3 & 5 & 6 & 5 & 5 & 5 & 5 & 4 \\
\hline$\frac{1}{512}$ & 4 & 3 & 5 & 6 & 6 & 6 & 5 & 5 & 5 \\
\hline
\end{tabular}
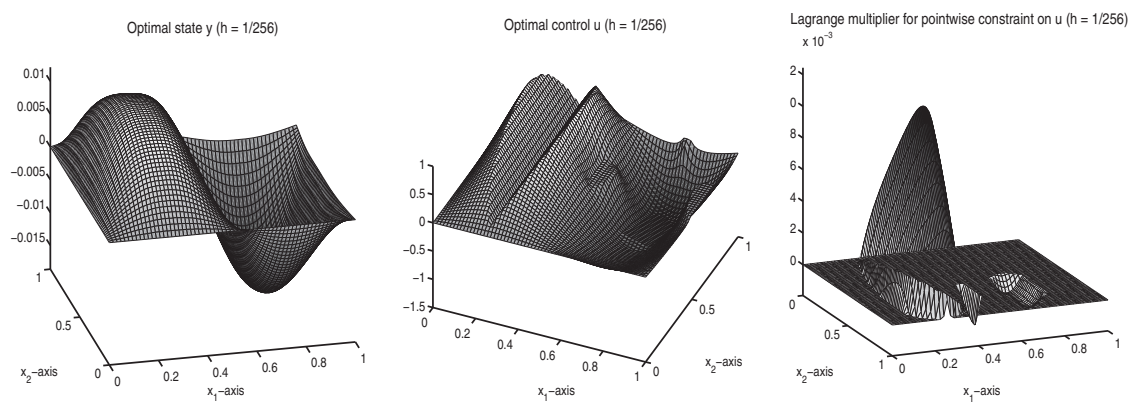

FIG. 4.3. Problem $2(\gamma=1 E 8, h=1 / 256)$. State $y_{h}$, control $u_{h}$, and multiplier $\mu_{h}$ upon termination of Algorithm 1.
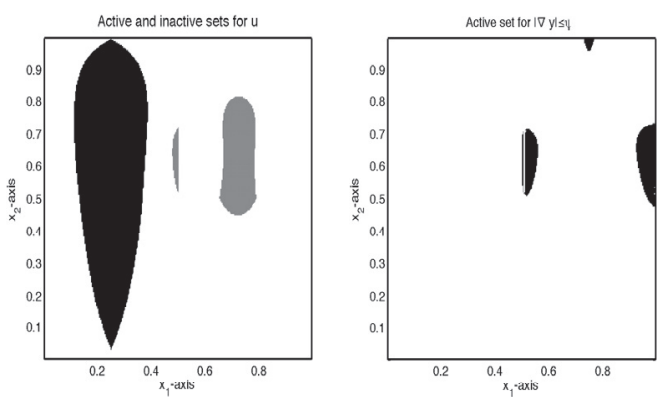

FIG. 4.4. Problem $2(\gamma=1 E 8, h=1 / 256)$. Inactive set (white), and active set for the lower bound (gray) and for the upper bound (black) in the left plot. Approximation of the active set for the first-order state constraint (black) in the right plot.

and the approximation for the active set for the pointwise gradient-constraint on the state on the right. As before, Table 4.2 provides the iteration numbers upon successful termination for various mesh sizes and $\gamma$-values.

Concerning the mesh independence and the stability of the iteration counts due to the employed $\gamma$-continuation scheme, the results of Table 4.2 support the same conclusions as for Problem 1. Again, without the continuation technique Algorithm 1 may fail to converge for the simple initial guess $u_{h}^{0}=0$. We observe that a stable (with respect to $h$ and $\gamma$ ) and a superlinear convergence behavior of the semismooth Newton method is obtained without utilizing the lifting step.

Next we demonstrate the difference in regularity between $\lambda_{\gamma}$ of Problem 1 (see Figure 4.5; left plot) and $\lambda_{\gamma}^{s}$ of Problem 2 (see Figure 4.5; right plot). Note that for visualization purposes we linearly interpolate the multiplier values at the grid 
TABLE 4.2

Problem 2. Number of iterations for various mesh sizes and $\gamma$-values.

\begin{tabular}{|c||c|c|c|c|c|c|c|c|c|}
\hline \multicolumn{10}{|c|}{ Iterations } \\
\hline$h / \gamma$ & $1 \mathrm{E} 0$ & $1 \mathrm{E} 1$ & $1 \mathrm{E} 2$ & $1 \mathrm{E} 3$ & $1 \mathrm{E} 4$ & $1 \mathrm{E} 5$ & $1 \mathrm{E} 6$ & $1 \mathrm{E} 7$ & $1 \mathrm{E} 8$ \\
\hline \hline$\frac{1}{32}$ & 6 & 6 & 6 & 4 & 3 & 3 & 2 & 2 & 2 \\
\hline$\frac{1}{64}$ & 7 & 7 & 6 & 4 & 4 & 3 & 3 & 2 & 2 \\
\hline$\frac{1}{128}$ & 7 & 7 & 6 & 5 & 5 & 4 & 3 & 2 & 2 \\
\hline$\frac{1}{256}$ & 7 & 7 & 6 & 6 & 5 & 5 & 4 & 3 & 2 \\
\hline
\end{tabular}
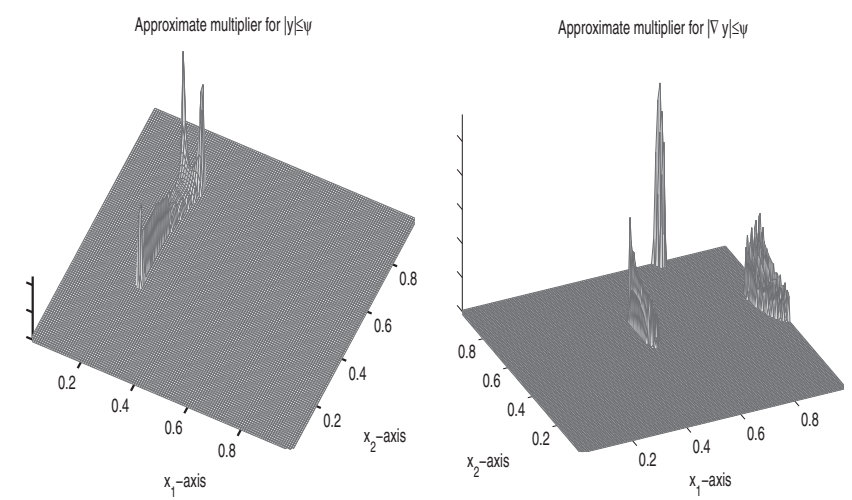

FIG. 4.5. $\gamma=1 E 8, h=1 / 256$. Approximate multiplier $\lambda_{\gamma, h}$ for Problem 1 (left) and $\lambda_{\gamma, h}^{s}$ for Problem 2 (right).

points. The approximate multiplier $\lambda_{\gamma, h}$ reflects the structural result obtained in [2]. According to this result, under sufficient regularity the multiplier is $L^{2}$-regular on the active set, zero on the inactive set, and measure-valued on the boundary between the active and inactive set. Such a structure can be observed by inspecting the left plot of Figure 4.5. On the other hand, for pointwise state constraints of gradienttype (first-order constraints) additional regularity on the active set appears not to be available for the example under investigation. Indeed, $\lambda_{\gamma, h}^{s}$ in the right plot of Figure 4.5 exhibits low regularity in the interior of the (smooth) active set.

Finally we note that the rather small value for tol and the rather large values for $\gamma$ in our numerics reflect our interest of studying Algorithm 1 as a solver for a given discrete problem. In view of the error in discretization, however, when solving $(\mathrm{P})$ by a sequence of approximating problems $\left(\mathrm{P}_{\gamma}\right)$ one would be interested in estimating the overall error in terms of the discretization and the $\gamma$-relaxation error, respectively. Such a result would allow a $\gamma$-choice such that both errors are balanced on a given mesh. This kind of numerical analysis is important in its own right, but goes beyond the scope of the present paper and is the subject of future research.

Appendix A. Auxiliary result. The following proposition establishes the Newton differentiability of a superposition of Newton differentiable maps.

Proposition A.1. Let $f: \mathcal{Y} \rightarrow \mathcal{Z}$ and $g: \mathcal{X} \rightarrow \mathcal{Y}$ be Newton differentiable in open sets $V$ and $U$, respectively, with $U \subset \mathcal{X}, g(U) \subset V \subset \mathcal{Y}$. Assume that $g$ is locally Lipschitz continuous and that there exists a Newton map $G_{f}(\cdot)$ of $f$ which is bounded on $g(U)$. Then the superposition $f \circ g: \mathcal{X} \rightarrow \mathcal{Z}$ is Newton differentiable in $U$ with a Newton map $G_{f} G_{g}$. 
Proof. Let $x \in U$ and consider

$$
\begin{aligned}
& \left|f(g(x+h))-f(g(x))-G_{f}(g(x+h)) G_{g}(x+h) h\right|_{\mathcal{Z}} \\
& =\left|f(w+k)-f(w)-G_{f}(w+k) k+R(x, h)\right|_{\mathcal{Z}},
\end{aligned}
$$

where $w=g(x), k=k(h)=g(x+h)-g(x)$ and $R(x, h)=G_{f}(g(x+h))(g(x+h)-$ $g(x))-G_{f}(g(x+h)) G_{g}(x+h) h$. Observe that

$$
\begin{aligned}
|R(x, h)|_{\mathcal{Z}} & =\left|G_{f}(g(x+h))\left(g(x+h)-g(x)-G_{g}(x+h) h\right)\right|_{\mathcal{Z}} \\
& \leq C\left|g(x+h)-g(x)-G_{g}(x+h) h\right|_{\mathcal{Y}}=\mathcal{O}\left(|h|_{\mathcal{X}}\right)
\end{aligned}
$$

as $|h|_{\mathcal{X}} \rightarrow 0$ by Newton differentiability of $g$ at $x$. Further, owing to the local Lipschitz continuity of $g$ there exists a constant $L>0$ such that $|g(x+h)-g(x)|_{\mathcal{Y}} \leq L|h|_{\mathcal{X}}$ for all $h$ sufficiently small. Hence, $|k(h)|_{\mathcal{Y}}=\mathcal{O}\left(|h|_{\mathcal{X}}\right)$ as $|h|_{\mathcal{X}} \rightarrow 0$. Now we continue (A.1) by

$$
\begin{aligned}
\mid f(w+k) & -f(w)-G_{f}(w+k) k+\left.R(x, h)\right|_{\mathcal{Z}} \\
& \leq\left|f(w+k)-f(w)-G_{f}(w+k) k\right|_{\mathcal{Z}}+\mathcal{O}\left(|h|_{\mathcal{X}}\right) \\
& =\mathcal{O}\left(|k|_{\mathcal{Y}}\right)+\mathcal{O}\left(|h|_{\mathcal{X}}\right)=\mathcal{O}\left(|h|_{\mathcal{X}}\right)
\end{aligned}
$$

as $|h|_{\mathcal{X}} \rightarrow 0$, where we use the Newton differentiability of $f$ at $g(x)$. This proves the assertion.

\section{REFERENCES}

[1] N. Arada And J.-P. Raymond, Dirichlet boundary control of semilinear parabolic equations. I. Problems with no state constraints, Appl. Math. Optim., 45 (2002), pp. 125-143.

[2] M. Bergounioux and K. Kunisch, On the structure of Lagrange multipliers for stateconstrained optimal control problems, Systems Control Lett., 48 (2003), pp. 169-176.

[3] C. Büskens and H. Maurer, SQP-methods for solving optimal control problems with control and state constraints: Adjoint variables, sensitivity analysis and real-time control, J. Comput. Appl. Math., 120 (2000), pp. 85-108.

[4] E. CASAS, Control of an elliptic problem with pointwise state constraints, SIAM J. Control Optim., 24 (1986), pp. 1309-1318.

[5] E. Casas And L. A. Fernandez, Optimal control of semilinear equations with pointwise constraints on the gradient of the state, Appl. Math. Optim., 27 (1993), pp. 35-56.

[6] E. Casas, F. Tröltzsch, AND A. Unger, Second order sufficient optimality conditions for a class of elliptic control problems, in Control and Partial Differential Equations (MarseilleLuminy, 1997), Vol. 4 of ESAIM Proc., Soc. Math. Appl. Indust., Paris, 1998, pp. 285-300.

[7] X. Chen, Z. NAShed, AND L. QI, Smoothing methods and semismooth methods for nondifferentiable operator equations, SIAM J. Numer. Anal., 38 (2000), pp. 1200-1216.

[8] M. Hintermüller, K. Ito, And K. KUnisCh, The primal-dual active set strategy as a semismooth Newton method, SIAM J. Optim., 13 (2003), pp. 865-888.

[9] M. Hintermüller and K. Kunisch, Feasible and non-interior path-following in constrained minimization with low multiplier regularity, SIAM J. Control Optim., 45 (2006), pp. 11981221.

[10] M. Hintermüller And K. Kunisch, Path-following methods for a class of constrained minimization problems in function space, SIAM J. Optim., 17 (2006), pp. 159-187.

[11] M. Hintermüller And W. RING, Numerical aspects of a level set based algorithm for state constrained optimal control problems, Comput. Assisted Mech. Eng. Sci., 3 (2003), pp. 149161.

[12] M. Hintermüller And W. RING, A level set approach for the solution of a state constrained optimal control problem, Numer. Math., (2004), pp. 135-166.

[13] K. Iто AND K. KUNISCH, Lagrange multiplier approach to variational problems and applications, Advances in Design and Control, SIAM, Philadelphia, 2008.

[14] K. Iто And K. Kunisch, Semi-smooth Newton methods for state-constrained optimal control problems, Systems Control Lett., 50 (2003), pp. 221-228.

Copyright (c) by SIAM. Unauthorized reproduction of this article is prohibited. 
[15] K. Krumbiegel and A. Rösch, On the regularization error of state constrained Neumann control problems, Control Cybernet., 37 (2008), pp. 369-392.

[16] P. Lu, Non-linear systems with control and state constraints, Optimal Control Appl. Methods, 18 (1997), pp. 313-326.

[17] C. Meyer, A. Rösch, And F. Tröltzsch, Optimal control of PDEs with regularized pointwise state constraints, Comput. Optim. Appl., 33 (2006), pp. 209-228.

[18] R. Mifflin, Semismooth and semiconvex functions in constrained optimization, SIAM J. Control Optim., 15 (1977), pp. 959-972.

[19] P. Neittaanmäki, J. Sprekels, and D. Tiba, Optimization of Elliptic Systems, Springer, Berlin, 2006.

[20] U. Prüfert, F. Tröltzsch, AND M. Weiser, The convergence of an interior point method for an elliptic control problem with mixed control-state constraints, Comput. Optim. Appl., 39 (2008), pp. 183-218.

[21] L. QI AND J. Sun, A nonsmooth version of Newton's method, Math. Program. Ser. A, 58 (1993), pp. 353-367.

[22] J. P. RAYMOND, Optimal control problem for semilinear parabolic equations with pointwise state constraints, in Modelling and Optimization of Distributed Parameter Systems (Warsaw, 1995), Chapman \& Hall, New York, 1996, pp. 216-222.

[23] J. P. RAYmond, Nonlinear boundary control of semilinear parabolic problems with pointwise state constraints, Discrete Contin. Dyn. Syst., 3 (1997), pp. 341-370.

[24] J.-P. RAYmond And F. Tröltzsch, Second order sufficient optimality conditions for nonlinear parabolic control problems with state constraints, Discrete Contin. Dyn. Syst., 6 (2000), pp. 431-450.

[25] D. Tiba And C. Zalinescu, On the necessity of some constraint qualification conditions in convex programming, J. Convex Anal., 11 (2004), pp. 95-110.

[26] G. M. Troinniello, Elliptic Differential Equations and Obstacle Problems, The University Series in Mathematics. Plenum Press, New York, 1987.

[27] F. TrÖLTZSCH, Regular Lagrange multipliers for control problems with mixed pointwise controlstate constraints, SIAM J. Optim., 15 (2005), pp. 616-634.

[28] M. Ulbrich, Semismooth Newton methods for operator equations in function spaces, SIAM J. Optim., 13 (2003), pp. 805-842.

Copyright (C) by SIAM. Unauthorized reproduction of this article is prohibited. 